TRANSACTIONS OF THE

AMERICAN MATHEMATICAL SOCIETY

Volume 362, Number 9, September 2010, Pages 4695-4734

S 0002-9947(10)04993-7

Article electronically published on April 28, 2010

\title{
STRUCTURE OF ZARISKI-CLOSED ALGEBRAS
}

\author{
ALEXEI BELOV-KANEL, LOUIS ROWEN, AND UZI VISHNE
}

\begin{abstract}
The objective of this paper is to describe the structure of Zariskiclosed algebras, which provide a useful generalization to finite dimensional algebras in the study of representable algebras over finite fields. Our results include a version of Wedderburn's principal theorem as well as a more explicit description using representations, in terms of "gluing" in Wedderburn components. Finally, we construct "generic" Zariski-closed algebras, whose description is considerably more complicated than the description of generic algebra of finite dimensional algebras.

Special attention is given to infinite dimensional algebras over finite fields.
\end{abstract}

\section{INTRODUCTION}

This paper grew out of work on algebras satisfying a polynomial identity (PI). We recall [BR, pp. 28ff.] that a PI-algebra $R$ over an integral domain $C$ is representable if it can be embedded as a subalgebra of $\mathrm{M}_{n}(K)$ for a suitable field $K \supset C$ (which can be much larger than $C$ ). One main byproduct of Kemer's theorem BR, Corollary 4.67] is that every relatively free affine PI-algebra over an infinite field is representable. From this perspective, the proof of Kemer's theorem is based on a close study of representable algebras. The strategy is to find the PI-algebra with the "best" structure, that is PI-equivalent to a given representable algebra, in order to study its identities very carefully. (Note that in characteristic 0 for the non-affine case, Kemer proved that any relatively free algebra can be embedded in the Grassmann envelope of a finite dimensional superalgebra, so similar considerations also hold in this case.)

Whereas over an infinite field, any representable algebra is PI-equivalent to a finite dimensional $K$-algebra (thus leading to a very careful study of identities of finite dimensional algebras in the proof of Kemer's theorem), this is no longer the case over finite fields (in positive characteristic). Thus, we need to replace finite dimensional algebras by a more general class, called Zariski-closed algebras, which, surprisingly, satisfy much of the structure theory of finite dimensional algebras. Since the relatively free affine algebra of an affine PI-algebra is representable, we are led finally to study the Zariski closure of a (representable) relatively free algebra.

Received by the editors September 22, 2008.

2010 Mathematics Subject Classification. Primary 16G99.

This research was supported by the Israel Science Foundation, grant \#1178/06.

(C)2010 American Mathematical Society Reverts to public domain 28 years from publication 
Throughout the paper, $F \subseteq K$ will be fields, with $F$ finite or infinite and $K$ usually being algebraically closed; $A$ is an $F$-algebra contained in a finite dimensional $K$-algebra $B$. We usually assume that $F$ has characteristic $p>0$, since the theory becomes standard in characteristic 0 .

After some introductory comments in Section 2, we introduce the Zariski closure of a representable algebra $A$ in Section 3 showing that it shares many of the important structure theorems of finite dimensional algebras, such as Wedderburn's principal theorem and the fact that every semiprime Zariski-closed algebra is semisimple; it turns out that Zariski-closed algebras are semiperfect. Identities and defining relations of $A$ also are studied in terms of its Zariski closure in $B$, to be defined below.

In Section 4 we delve more deeply into the generation of polynomial relations of a Zariski-closed algebra, showing that the center is defined in terms of finitely many polynomial relations, which can be written in the form $\lambda_{i}=0, \lambda_{i}-\lambda_{i}^{s}=$ 0 , where $s$ is a $p$-power, or $\lambda_{i}-\lambda_{j}^{s}=0, j \neq i$, where $s$ is a $p$-power. These polynomial relations are said to be of Frobenius type. This enables us explicitly to study representations of Zariski-closed algebras in Section [5] focusing on their Peirce decomposition, and its refinements. The explicit representation of algebras is complicated even in characteristic 0 , and one of our main techniques is "gluing,"

or identifying different components in a representation.

In Section 6, we also obtain results concerning the off-diagonal polynomial relations, which requires us to consider $q$-polynomials, which we call polynomial relations of weak Frobenius type. The main result is that the weak Frobenius relations comprise a free module over the group algebra of the Frobenius automorphism. We thank B. Kunyavskii for bringing to our attention the references [KMT], [M], and $[\mathrm{T}$.

Finally, in Section 7 we describe the relatively free algebras of Zariski-closed algebras. These turn out to have an especially nice description and play a key role in the proof of Specht's conjecture for affine PI-algebras of arbitrary characteristic.

\section{BACKGROUND}

Let us bring in the main tools for our study.

2.1. Results from the theory of finite dimensional algebras. We start with a classical theorem of Wedderburn about finite dimensional algebras:

Theorem 2.1 (Wedderburn's Principal Theorem). Any finite dimensional algebra $A$ over a perfect field $F$ has a Wedderburn decomposition $A=S \oplus J$, where $J$ is the Jacobson radical of $A$, which in this case is also the largest nilpotent ideal, and $S \cong A / J$ is a semisimple subalgebra of $A$.

When the base field is algebraically closed, Wedderburn's Principal Theorem enables us to find a direct product of matrix rings inside any finite dimensional algebra $A$. The following notion helps us to better understand the structure of $A$.

We call $\left\{e_{1}, \ldots, e_{n}\right\}$ a 1-sum set of orthogonal idempotents if they are orthogonal and $\sum_{i=1}^{n} e_{i}=1$. 
Remark 2.2 ("Peirce decomposition"). If $A$ has a $\mathbf{1}$-sum set of orthogonal idempotents $\left\{e_{1}, \ldots, e_{n}\right\}$ (i.e., $e_{i} e_{j}=0$ for all $i \neq j$ ), then

$$
A=\bigoplus_{i, j=1}^{t} e_{i} A e_{j}
$$

as additive groups.

For example, the Peirce decomposition of $A=\mathrm{M}_{n}(R)$ with respect to the matrix units $e_{11}, e_{22}, \ldots, e_{n n}$ is just

$$
A=\bigoplus_{i, j=1}^{n} A e_{i j}
$$

Note that any set $\left\{e_{1}, \ldots, e_{n}\right\}$ of orthogonal idempotents of $A$ can be expanded to a 1 -sum set $\left\{e_{0}, e_{1}, \ldots, e_{n}\right\}$ by taking $e=\sum_{i=1}^{n} e_{i}$ and putting $e_{0}=1-e$.

Even for algebras without 1, one can reproduce an analog of the Peirce decomposition by formally defining a left and right operator $e_{0}$ from $A$ to $A$, given by

$$
e_{0} a=a-e a, \quad a e_{0}=a-a e .
$$

2.2. Affine varieties and algebraic groups. We need some basic facts from affine algebraic geometry and the theory of affine algebraic groups. We use $[\mathrm{H}]$ as a reference for algebraic groups. Suppose $K$ is an algebraically closed field. Write $K[\Lambda]$ for the polynomial algebra $K\left[\lambda_{1}, \ldots, \lambda_{n}\right]$. For any subset $S \subset K[\Lambda]$, we define the zero set of $S$ to be

$$
\mathcal{Z}(S)=\left\{\mathbf{a}=\left(\alpha_{1}, \ldots, \alpha_{n}\right) \in K^{(n)}: f\left(\alpha_{1}, \ldots, \alpha_{n}\right)=0, \forall f \in S\right\} .
$$

$K^{(n)}$ has the Zariski topology whose closed sets are the zero sets of subsets of $K[\Lambda]$. This is the smallest topology under which all polynomial maps $K^{(n)} \rightarrow K$ are continuous, assuming $K$ has the co-finite topology.

A closed set is irreducible if it is not the union of two proper closed subsets. An affine variety is a Zariski-closed subset of $K^{(n)}$. The dimension of a variety is the length of a maximal chain of irreducible subvarieties, with respect to (proper) inclusion. A morphism of varieties is a continuous function with respect to the respective topologies. (In this text we concern ourselves only with affine varieties, so "variety" means "affine variety.")

A locally closed set is the intersection of a closed set and an open set. A constructible set is the finite union of locally closed sets. We need the following theorem of Chevalley:

Theorem 2.3 ([ $\mathrm{H}$, Theorem 4.4]). Any morphism of varieties sends constructible sets to constructible sets. (In particular, the image of a variety is constructible.)

An (affine) algebraic group is an (affine) variety $G$ endowed with a group structure $(G, \cdot, e)$ such that the inverse operation (given by $g \mapsto g^{-1}$ ) and multiplication map $G \times G \rightarrow G$ (given by $(a, b) \mapsto a \cdot b)$ are morphisms of varieties. A morphism $\varphi: G \rightarrow H$ of algebraic groups is a group homomorphism that is also a morphism of varieties.

Theorem 2.4 ([ $\mathrm{H}$, Proposition 7.3]). In any algebraic group $G$, the irreducible component $G_{e}$ of the identity is a closed connected subgroup of finite index, whose cosets are precisely the (connected) irreducible components of $G$. Thus, as a variety, $G$ is the direct product of an irreducible variety and a finite set. 
By [H, Theorem 11.5], for any affine algebraic group $G$ with closed normal subgroup $N$, the group $G / N$ can be provided with the structure of an algebraic group.

2.3. Frobenius automorphisms and finite fields. Much of our theory depends on the properties of endomorphisms of finite fields. Towards this end, we recall the Frobenius endomorphism of a field $F$ of characteristic $p$ given by $a \mapsto a^{p^{t}}$ for suitable fixed $t$. When $F$ is finite, then every algebra endomorphism of $F$ is obviously an automorphism (over its characteristic subfield), and it is well known by Galois theory that every automorphism of $F$ is Frobenius.

When $F$ is an infinite field, there may of course be non-Frobenius endomorphisms (but one can show using a Vandermonde matrix argument, for any automorphism $\sigma$, that if $\sigma(a)$ and $a$ are algebraically dependent of bounded degree for all $a \in F$, then $\sigma$ is a Frobenius endomorphism).

Note that the Frobenius endomorphism of an algebraically closed field $K$ also is an automorphism of $K$, although $K$ is infinite.

Theorem 2.5 (Wedderburn's theorem about finite division rings). Any finite division ring is commutative. Consequently, any finite dimensional simple algebra over a finite field $F$ must have the form $\mathrm{M}_{n}\left(F_{1}\right)$ for a finite extension $F_{1}$ of $F$.

Any finite field $F$ can be viewed as the zero set of the polynomial $\lambda^{q}-\lambda$ in its algebraic closure $K$, where $q=|F|$. This observation enables us to view finite fields explicitly as subvarieties (of dimension 0) of the affine line. Likewise, matrices over finite fields can be viewed naturally as varieties.

2.4. Examples of representable PI-algebras over finite and infinite fields. A polynomial identity (PI) of an algebra $A$ is a polynomial which vanishes identically for any substitution in $A$. Recall that a ring $R$ is called a central extension of a subring $A$ if $R=\operatorname{Cent}(R) A$. If $A$ is an algebra over an infinite field, then any central extension of $A$ is PI-equivalent to $A$; cf. [R1, Proposition 1.1.32]. Thus, in the examples to follow, the finiteness of the field $F$ is crucial for their special properties concerning identities.

Example 2.6. Suppose $F \subseteq K$ are fields.

(1) Let $A=\left(\begin{array}{cc}F & K \\ 0 & F\end{array}\right)$ (which is an $F$-algebra but not a $K$-algebra). Then $\left(\begin{array}{cc}K & K \\ 0 & K\end{array}\right)$ is a central extension of $A$ since $A$ contains the matrix units $e_{11}, e_{12}$, and $e_{22}$. When $F$ is infinite, $A$ is PI-equivalent to $\left(\begin{array}{cc}K & K \\ 0 & K\end{array}\right)$. However, when $|F|=q$ is finite, then $\alpha^{q}=\alpha$ for all $\alpha \in F$, implying $a^{q}-a \in\left(\begin{array}{cc}0 & K \\ 0 & 0\end{array}\right)$ for $a \in A$. Hence $\left(x^{q}-x\right)\left(y^{q}-y\right) \in \operatorname{id}(A)$.

(2) Let $A=\left(\begin{array}{ll}F & K \\ 0 & K\end{array}\right)$, where $|F|=q$. Then $a^{q}-a \in\left(\begin{array}{ll}0 & K \\ 0 & K\end{array}\right)$, for all $a \in A$, implying $\left(x^{q}-x\right)[y, z] \in \operatorname{id}(A)$.

(3) Let $A=\left(\begin{array}{cc}K & K \\ 0 & F\end{array}\right)$, where $|F|=q$. Then, analogously to (2), $[y, z]\left(x^{q}-x\right) \in$ $\operatorname{id}(A)$.

There is another type of example, involving identification of elements. 
Example 2.7. Suppose $\sigma$ is an automorphism of $F_{1}$ over $F$, where $F \subseteq F_{1} \subseteq K$. Then $K$ can be viewed as an $F_{1}$-left module in the usual way and as a right module "twisted" by $\sigma$; namely $a \cdot \alpha$ is defined as $a \sigma^{-1}(\alpha)$ for $a \in K, \alpha \in F$. (We denote this new right module structure as $\left.K_{\sigma}.\right)$ Then $\left(\begin{array}{cc}F_{1} & K_{\sigma} \\ 0 & F_{1}\end{array}\right)$ is a PI-algebra, which is clearly isomorphic to $\left(\begin{array}{cc}F_{1} & K \\ 0 & F_{1}\end{array}\right)$ as a ring (but not as an $F$-algebra). However, we get interesting new examples by making certain identifications.

(1) Suppose $\left|F_{1}\right|=q^{t}$, where $|F|=q$. Then we have the Frobenius automorphism $\alpha \mapsto \alpha^{q^{n}}$ of $F_{1}$, and $\left\{\left(\begin{array}{cc}\alpha^{p^{n}} & a \\ 0 & \alpha\end{array}\right): \alpha \in F_{1}, a \in K\right\}$ satisfies the identity $x[y, z]=[y, z] x^{p^{n}}$. Note that this $F$-algebra is not an $F_{1}$-algebra in general.

(2) Let $A=\left\{\left(\begin{array}{cc}\sigma(\alpha) & a \\ 0 & \alpha\end{array}\right): \alpha \in F_{1}, a \in K\right\}$. As a consequence of Theorem 4.10 to be proved below, if $\sigma$ is not Frobenius, then $\operatorname{id}(A)=\operatorname{id}\left(T_{2}\right)$, where $T_{2}$ is the algebra of $2 \times 2$ triangular matrices.

We call this identification process gluing, and it will be described more precisely in Section 5 . All of Example 2.6 and Example 2.7 have a central extension to $B=\left(\begin{array}{cc}K & K \\ 0 & K\end{array}\right)$, and thus they satisfy the same multilinear identities of $K$. But these varieties are quite different. Thus, as opposed to algebras over infinite fields, in general the multilinear identities are far from describing the full PI picture.

For later use, we record the following result.

Proposition 2.8. If $A=A_{1}+A_{2}$, then a non-commutative polynomial $f$ is an identity of $A$ iff $f$ and its consequences become zero under substitutions in which every variable takes values either in $A_{1}$ or in $A_{2}$.

Proof. This is trivial is characteristic zero, where every identity is equivalent to a set of multilinear ones. In general, the proof is by induction on the degree of $f$, considering the multilinearization $f(\vec{x}+\vec{y})-f(\vec{x})-f(\vec{y})$.

\section{The Zariski closure of a representable algebra}

Both the motivation for PI-theory and one of its major facets is the theory of representable algebras. In this section we develop this theory, with emphasis always on the set of identities of a given representable algebra $A$. Thus we often exchange $A$ by an appropriate PI-equivalent algebra.

Let $F$ be a field and $A$ an arbitrary $F$-algebra. Recall from the introduction that $A$ is representable if $A$ embeds (as an $F$-algebra) in $\mathrm{M}_{n}(K)$ for a suitable extension field $K$ (possibly infinite dimensional) of $F$ and a suitable $n$. In this section we assume throughout that $A$ is representable. Then $A$ can be embedded further in $B=\mathrm{M}_{n}(\bar{K})$, where $\bar{K}$ is the algebraic closure of $K$, so we assume throughout, without loss of generality, that $K$ is algebraically closed. Thus, we view $\mathrm{M}_{n}(K)$ as an $n^{2}$-dimensional variety and have the theory of affine algebraic geometry at our disposal.

When the base field $F$ is infinite, $A$ is PI-equivalent to the $K$-subalgebra $K A$ of $\mathrm{M}_{n}(K)$, which is finite dimensional, so one passes at once to the finite dimensional 
case over an algebraically closed field. In other words, one considers finite dimensional algebras over a field, in which case one has the tools from the theory of finite dimensional algebras, as described above.

However, over finite fields (which clearly have positive characteristic), it does not suffice to consider $K$-subalgebras of $\mathrm{M}_{n}(K)$, as evidenced in Example 2.6, where we have examples of algebras $A$ for which $K A=\left(\begin{array}{cc}K & K \\ 0 & K\end{array}\right)$, but $A$ satisfies extra identities. Thus we need a subtler way, not passing all the way to the algebraic closure, of obtaining "canonical" algebras that are PI-equivalent to a given representable algebra.

Our solution is to consider the Zariski closure of $A$ in $\mathrm{M}_{n}(K)$, which enjoys the analogs of all of the properties of finite dimensional algebras listed above.

To show that an $F$-algebra $A$ is representable, it clearly is enough to embed $A$ into any finite dimensional unital $K$-algebra $B$, since letting $n=[B: K]$ we can further embed $B$ into $\mathrm{M}_{n}(K)$. So we consider this situation that $A \subseteq B$, where $B$ is an $n$-dimensional algebra over the algebraically closed field $K$. At first, we assume that $B$ is a matrix algebra, but later we modify our choice of $B$ to better reflect the structure of $A$.

\subsection{The Zariski closure.}

Definition 3.1. Suppose $B$ is a $K$-vector space, with $[B: K]=n$. Picking a base $b_{1}, \ldots, b_{n}$ of $B$ over $K$, we view $B$ as the affine variety $\mathbf{A}^{n}$ of dimension $n$, identifying an element $\sum_{i=1}^{n} \alpha_{i} b_{i}\left(\alpha_{i} \in K\right)$ with the vector $\left(\alpha_{1}, \ldots, \alpha_{n}\right)$. Usually $B$ is a $K$-algebra, but we formally do not need this requirement.

Suppose $F$ is a subfield of $K$ and $V \subset B$ is a vector space over $F$. The Zariski closure of $V$ inside $B$, denoted by $V_{B}^{\mathrm{cl}}$, is the closure of $V$ inside $B$ via the Zariski topology of $\mathbf{A}^{n}$ (identifying $B$ with $\mathbf{A}^{n}$ ). When $B$ is understood, we write $V^{\text {cl }}$ for $V_{B}^{\mathrm{cl}}$.

Recall that the Zariski topology of the affine variety $\mathbf{A}^{n}$ over $K$ is defined as having its closed sets be precisely those sets of simultaneous zeros of polynomials from the (commutative) polynomial algebra $K\left[\lambda_{1}, \ldots, \lambda_{n}\right]$. In other words, a closed subspace of $B$ can be defined by (finitely many) polynomials.

Remark 3.2. When we fix a base $b_{1}, \ldots, b_{n}$ for $B$, any polynomial $f \in K\left[\lambda_{1}, \ldots, \lambda_{n}\right]$ can be viewed as a function $f: B \rightarrow K$ by assigning

$$
f\left(\alpha_{1} b_{1}+\cdots+\alpha_{n} b_{n}\right)=f\left(\alpha_{1}, \ldots, \alpha_{n}\right) .
$$

A polynomial $f\left(\lambda_{1}, \ldots, \lambda_{n}\right)$ is called a polynomial relation on $A$ if $f(A)=0$, in the sense of Remark 3.2. Thus a polynomial relation $f\left(\lambda_{1}, \ldots, \lambda_{n}\right)$ is always taken in $\leq n$ indeterminates, and we check it by evaluating it on the coordinates of a single element $a$, for each $a$ in $A$. In contrast, in PI-theory, a polynomial identity $g\left(x_{1}, \ldots, x_{m}\right)$ of $A$ (resp. of $B$ ) can be in any number of indeterminates, specialized to $m$ elements of $A$ (resp. of $B$ ).

Remark 3.3. The Zariski closure does not depend on the choice of base of $B$ over $K$, since a linear transformation induces an automorphism of the polynomial ring (i.e., sends polynomial relations to polynomial relations) and thus does not change the Zariski topology. 
The Zariski closure does depend on the way in which $V$ is embedded in $B$ as an $F$-space, even for $F$ infinite. In particular, for an $F$-algebra $A$ contained in a $K$ algebra $B$, the notation $A_{B}^{\mathrm{cl}}$ should also indicate the particular representation of $A$ into $B$, as evidenced in the following example. (But nevertheless, the representation is usually understood, and so is not spelled out in the notation.)

Example 3.4. For $F=\mathbb{R}, K=\mathbb{C}$, and $B=\mathrm{M}_{n}(\mathbb{C})$, we could embed $A=\mathbb{C}$ into $M_{2}(\mathbb{C})$ as scalar matrices. On the other hand, in the spirit of Example 2.7. we could identify $\mathbb{C}$ with $\left\{\left(\begin{array}{ll}\alpha & 0 \\ 0 & \bar{\alpha}\end{array}\right): \alpha \in \mathbb{C}\right\}$, where ${ }^{-}$denotes the usual complex conjugation. In the first case, the Zariski closure of $A$ is $A$ itself, which is isomorphic to $\mathbb{C}$. In the second case, the Zariski closure of $A$ is $\left(\begin{array}{ll}\mathbb{C} & 0 \\ 0 & \mathbb{C}\end{array}\right) \cong \mathbb{C} \times \mathbb{C}$, which has larger dimension!

Although in this example $A \cong \mathbb{C}$ and thus $A$ is a $\mathbb{C}$-algebra, it is not Zariskiclosed in $M_{2}(\mathbb{C})$. Thus, $\mathbb{C}$ need not be Zariski-closed in $M_{2}(\mathbb{C})$ as an $\mathbb{R}$-algebra. But note here that $A$ is not a $\mathbb{C}$-subalgebra of $M_{2}(\mathbb{C})$, and in fact we have the following remark.

Remark 3.5. Any $K$-subspace $V$ of $B$ is Zariski-closed. (In particular, any $K$ subalgebra of $B$ is Zariski-closed.) Indeed, a $K$-subspace is an algebraic subvariety, defined by linear relations.

In particular, we have:

Lemma 3.6. $A^{\text {cl }} \subseteq K A$ inside $B$.

Proof. We saw in Remark 3.5 that $K A$ is Zariski-closed. Thus, the Zariski closure $A^{\mathrm{cl}}$ of $A$ is always contained in $K A$.

Thus, we call $K A$ the linear closure of $A$.

Proposition 3.7. If $F$ is infinite, then the Zariski closure of an $F$-vector space $A$ is equal to the linear closure of $A$.

Proof. By definition, $A^{\text {cl }}$ is composed of the common zeros in $B$ of the polynomial relations of $A$. Let $a \in A$, and let $f \in K\left[\lambda_{1}, \ldots, \lambda_{n}\right]$ be a polynomial relation. Then $f(\alpha a)=0$ for every $\alpha \in F$; viewing $\alpha$ generically, we see that $f(\alpha a)$ is identically zero. Therefore, $f(\alpha a)=0$ for every $\alpha \in K$, which proves that $K a \subseteq A^{\mathrm{cl}}$.

\section{Remark 3.8.}

(1) If a vector space is Zariski-closed, then any subset defined by polynomial relations is Zariski-closed.

(2) If $V \subseteq B_{0} \subseteq B$, then the Zariski closure of $V$ in $B$ is equal to the Zariski closure of $V$ in $B_{0}$. (Indeed, $B_{0}$ is closed in $B$ by Remark 3.5.)

(3) Suppose $A_{i} \subseteq B_{i}$ for $i=1,2$, where $B=B_{1} \oplus B_{2}$. Then

$$
\left(A_{1}+A_{2}\right)_{B}^{\mathrm{cl}}=A_{1}^{\mathrm{cl}}+A_{B_{1}}^{\mathrm{cl}} \text {. }
$$

(Indeed, $\left(b_{1}, b_{2}\right) \in B_{1} \oplus B_{2}$ satisfies all the polynomial relations of $A_{1}+A_{2}$ iff the $b_{i}$ satisfy all polynomial relations of $A_{i}$, for $i=1,2$.)

The distinction between finite and infinite fields, which is crucial in what is to come, is explained by the following observation. 
Example 3.9. (1) If $F$ is an infinite subfield of $K$, then $F$ satisfies only the identities resulting from commutativity, and thus $F^{\mathrm{cl}}=K$ (this follows, e.g., from Proposition 3.7 below). On the other hand, if $F$ is a finite field of order $q$, then $\lambda^{q}-\lambda=0$ is an identity, and $F^{\mathrm{cl}}=F$.

(2) The Zariski closure of $A=\left(\begin{array}{cc}F & K \\ 0 & F\end{array}\right)$ in $M_{2}(K)$ is $A$ if $F$ is finite and $\left(\begin{array}{cc}K & K \\ 0 & K\end{array}\right)$ otherwise.

Example 3.10. If $A_{i}$ are subsets of $B$, then clearly $\left(\bigcap A_{i}\right)^{\mathrm{cl}} \subseteq \bigcap\left(A_{i}^{\mathrm{cl}}\right)$. However, this may not be an equality. Indeed, let $\mu$ be an indeterminate over $\mathbb{F}_{q}$, and take $A_{i}=\mathbb{F}_{p}\left(\mu^{i}\right)$ for $i \in \mathbb{N}$, as subalgebras of the common algebraic closure $K$. We have that $A_{i}^{\text {cl }}=K$ since these are infinite fields, where $\bigcap A_{i}=\mathbb{F}_{p}$, which is closed.

From now on, we assume that $B$ is a $K$-algebra.

\section{Theorem 3.11.}

(1) If $V$ is an $F$-subspace of $B$, then $V^{\mathrm{cl}}$ is also an $F$-subspace.

(2) If $A$ is an $F$-subalgebra of $B$, then $A^{\text {cl }}$ is also an $F$-subalgebra.

(3) If $I$ is a left ideal of $A$, then $I^{\mathrm{cl}}$ is a left ideal of $A^{\mathrm{cl}}$.

(4) If $I \triangleleft A$, then $I^{\mathrm{cl}} \triangleleft A^{\mathrm{cl}}$.

Proof. (1) Given any $a \in B$ and any polynomial relation $f$ vanishing on $A$, define $f_{a}(x)=f(a+x)$. Clearly, for each $a \in A, f_{a}$ vanishes on $A$, and thus on $A^{\mathrm{cl}}$, i.e. $f(a+r)=0$ for all $r \in A^{\mathrm{cl}}$. Thus, $f_{r}$ vanishes on $A$ for $r \in A^{\mathrm{cl}}$, implying $f_{r}$ vanishes on $A^{\mathrm{cl}}$, i.e., $f(r+s)=0$ for all $r, s \in A^{\mathrm{cl}}$. This is true for every $f$ vanishing on $A$, proving $r+s \in A^{\text {cl }}$; i.e., $A^{\text {cl }}$ is closed under addition.

Likewise, defining $(\alpha f)(x)=f(\alpha x)$, we see for each $\alpha \in F$ that $\alpha f$ vanishes on $A$ and thus on $A^{\text {cl }}$; i.e., $f(\alpha r)=0$ for all $r \in A^{\text {cl }}$, i.e., $A^{\text {cl }}$ is a $F$-vector space.

(2) Continuing the idea of (11), given any $a \in B$ and any polynomial relation $f$ vanishing on $A$, define $f_{a}(x)=f(a x)$. Then, for each $a \in A, f_{a}$ vanishes on $A$ and thus on $A^{\mathrm{cl}}$, implying $f_{a}(r)=0$ for all $r \in A^{\mathrm{cl}}$. Repeating this argument for $f_{r}$ shows that $f(r s)=0$ for all $r, s \in A^{\mathrm{cl}}$, and we conclude that $r s \in A^{\mathrm{cl}}$.

(3) By (11), $I^{\mathrm{cl}}$ is a subgroup of $A^{\mathrm{cl}}$. But for any $a \in A$ and any polynomial relation $f$ vanishing on $I$, we define $f_{a}(x)=f(a x)$, which also vanishes on $I$ and thus on $I^{\mathrm{cl}}$. Using the same trick and defining $f_{r}(x)=x r$, we see, for any $r \in I^{\mathrm{cl}}$, that $f_{r}$ vanishes on $A$ and thus on $A^{\mathrm{cl}}$, implying $A^{\mathrm{cl}} I^{\mathrm{cl}} \subseteq I^{\mathrm{cl}}$; i.e., $I^{\mathrm{cl}}$ is a left ideal of $A^{\mathrm{cl}}$.

(4) Also apply the right-handed version of (3).

The Zariski closure acts functorially and turns out to be a key tool in the structure of algebras. To see this, we need to show that the Zariski closure preserves various important structural properties. Sometimes it is convenient to separate addition from multiplication in our discussion. The Zariski closure of an additive subgroup $(G,+)$ of $\mathrm{M}_{n}(K)$ is a closed subgroup; i.e., an algebraic group.

Proposition 3.12 ([, Cor. 7.4]). Suppose $G$ is any algebraic group that comes with a morphism of algebraic groups $\psi: G \rightarrow V$. Then $\psi(G)$ is Zariski-closed in $V$.

Corollary 3.13. Suppose $A$ is a Zariski-closed algebra and $\psi: A \rightarrow B^{\prime}$ is a morphism of varieties. Then $\psi(A)$ is closed in $B^{\prime}$. 
Corollary 3.14. For every $F$-subalgebra $A$ of $B$ and morphism $\psi: B \rightarrow B^{\prime}, \psi\left(A^{\mathrm{cl}}\right)=$ $\psi(A)^{\mathrm{cl}}$.

Proof. Since $\psi\left(A^{\mathrm{cl}}\right)$ is closed, we have that $\psi(A)^{\mathrm{cl}} \subseteq \psi\left(A^{\mathrm{cl}}\right)$; but $\psi\left(A^{\mathrm{cl}}\right) \subseteq \psi(A)^{\mathrm{cl}}$ by continuity of $\psi$.

Thus, we see how the power of algebraic group techniques enters into the theory of Zariski-closed algebras. There is a newer theory of algebraic semigroups $\mathrm{P}$ that would also enable us to utilize the multiplicative structure; we return to this later.

Corollary 3.15. Let $W \subseteq B$ be $K$-spaces. For any closed $F$-subspace $A \subseteq B$, the factor space $A /(W \cap A)$ can be identified with a Zariski-closed subspace of $B / W$.

Proof. Letting $\psi: B \rightarrow B / W$ be the projection morphism, $A /(W \cap A) \cong(A+W) / W$ $=\psi(A)$ is closed by Corollary 3.14

Corollary 3.16. If $A$ is a Zariski-closed $F$-subalgebra of $B$ and $I \triangleleft B$, then $A /(I \cap A)$ can be identified with a Zariski-closed subalgebra of $B / I$.

Proof. A special case of Corollary 3.15.

\subsection{PI's versus polynomial relations.}

Proposition 3.17. The polynomial identities of the finite dimensional K-algebra $B$ are determined by the polynomial relations in the Zariski topology.

Proof. Fixing the base $\left\{b_{i}\right\}$, we can take any polynomial $f\left(x_{1}, \ldots, x_{m}\right)$ defined on $B$, and, for any $w_{2}, \ldots, w_{m} \in B$, define $\hat{f}\left(x_{1}\right)$ via $\hat{f}(b)=f\left(b, w_{2}, \ldots, w_{m}\right)$. Writing $b$ "generically" as $\sum \lambda_{i} b_{i}$ and $\hat{f}(b)=\sum \beta_{k} b_{k}$, we define $\hat{f}_{k}(b)=\beta_{k}$. Putting each $\beta_{k}=0$ in turn clearly defines a polynomial relation, since multiplication of the base elements of $B$ is given in terms of structure constants.

For example, suppose $b_{i} b_{j}=\sum \alpha_{i j k} b_{k}$ in $B$, and $f=x_{1} x_{2}-x_{2} x_{1}$. Fixing $w_{2}=\sum c_{i} b_{i}$, we have

$$
\hat{f}(b)=\sum \lambda_{i} b_{i} \sum c_{j} b_{j}-\sum c_{i} b_{i} \sum \lambda_{j} b_{j}=\sum_{k} \sum_{i, j} \alpha_{i j k}\left(c_{j} \lambda_{i}-c_{i} \lambda_{j}\right) b_{k},
$$

so, for each $k$,

$$
\hat{f}_{k}=\sum_{i, j} \alpha_{i j k}\left(c_{j} \lambda_{i}-c_{i} \lambda_{j}\right)
$$

In this way, letting $w_{2}, \ldots, w_{m}$ run over all elements of $B$, we can view any polynomial identity as an (infinite) aggregate of polynomial relations on the coefficients of the elements of $B$.

The converse is one of our main objectives: Can Zariski-closed algebras be differentiated by means of their polynomial identities? For example, any proper $K$ subalgebra of $\mathrm{M}_{n}(K)$ satisfies the Capelli identity $c_{n^{2}}$, which is not an identity of $\mathrm{M}_{n}(K)$ itself.

Although every multilinear identity of $A$ is also satisfied by $K A$, we may have $\operatorname{Var}(A) \neq \operatorname{Var}(K A)$ in non-zero characteristic. For example, if $A$ is the algebra of Example 2.7(1), then $x[y, z]=[y, z] x^{p^{n}}$ is an example of $A$ but not of $K A$. The pertinence of Zariski closure to PI-theory comes from the following obvious but crucial observation.

Lemma 3.18. $\operatorname{Var}_{\mathrm{F}}(A)=\operatorname{Var}_{\mathrm{F}}\left(A^{\mathrm{cl}}\right)$. 
Proof. By Proposition 3.17, any identity $f\left(x_{1}, \ldots, x_{m}\right)$ of $A$ can be described in terms of polynomial relations. Thus the polynomial identity $f$ passes to the Zariski closure $A^{\mathrm{cl}}$.

(The same proof shows that any generalized polynomial identity of $A$ remains a generalized polynomial identity of $A^{\mathrm{cl}}$; likewise for rational identities.)

Let us first consider polynomial identities when $F$ is infinite. Combining the lemma with Proposition 3.7, an $F$-subalgebra $A$ of $B$ is PI-equivalent to $K A$. Thus, up to PI-equivalence, when $F$ is infinite, the Zariski-closed $F$-algebras correspond precisely to the $K$-subalgebras of $\mathrm{M}_{n}(K)$, and we have nothing new.

On the other hand, non-isomorphic Zariski-closed algebras may be PI-equivalent. For example, the algebra of diagonal matrices $\left(\begin{array}{cc}K & 0 \\ 0 & K\end{array}\right)$ is PI-equivalent to the algebra of scalar matrices $\left\{\left(\begin{array}{ll}\alpha & 0 \\ 0 & \alpha\end{array}\right): \alpha \in K\right\}$. Nevertheless, the Zariski closure is a way of finding canonical representatives of varieties of PI-algebras, which becomes much more sensitive over finite fields.

3.3. The structure of Zariski-closed algebras. As promised in the Introduction, we now show that Zariski-closed algebras have a structure theory closely paralleling the structure of finite dimensional algebras over an algebraically closed field. Since we want to pass to the Zariski closure in order to find a "canonical" algebra PI-equivalent to $A$, we want this to be independent of the choice of $K$ algebra $B$ in which $A$ is embedded. But presumably $A$ could be embedded in two $K$-algebras $B_{1}$ and $B_{2}$, and could be Zariski-closed in $B_{1}$ but not in $B_{2}$. Towards this end, we say $A$ is maximally Zariski-closed if $A$ is Zariski-closed in $B$, and every non-zero ideal of $B$ intersects $A$ non-trivially.

Example 3.19. In general, a Zariski-closed algebra $A$ need not be maximally closed in $K A$. Indeed, let $A=\left\{\left(\begin{array}{cc}a & 0 \\ 0 & a^{p}\end{array}\right): a \in K\right\}$ where $p=\operatorname{Char} K$. Then $A$ is a field, but $K A=\left(\begin{array}{cc}K & 0 \\ 0 & K\end{array}\right)$ is not.

However, we have the following useful fact:

Proposition 3.20. Every Zariski-closed F-subalgebra $A$ in $B$ is maximally Zariskiclosed with respect to a suitable homomorphic image of $B$.

In particular, we may assume $A$ is maximally Zariski-closed in $K A$.

Proof. We proceed by induction on $\operatorname{dim}_{K} B$. If $A$ is not maximally Zariski-closed, then there is some ideal $I$ of $B$ maximal with respect to $I \cap A=0$. But then $A \subseteq B / I$ by Corollary 3.16. The second assertion follows by taking $B$ to be the $K$-space spanned by $A$, a property retained by homomorphic images.

For any subalgebra $A$ of a matrix algebra $\mathrm{M}_{n}(K)$, every nil ideal of $A$ is nilpotent, of nilpotence index bounded by $n$, by a theorem of Wedderburn; cf. R2, Theorem 2.6.31]. Thus, there is a unique largest nil (and thus nilpotent) ideal of $A$, which we write as $\operatorname{Rad}(A)$. Recall that $A$ is semiprime iff $\operatorname{Rad}(A)=0$.

Proposition 3.21. $\operatorname{Rad}\left(A^{\mathrm{cl}}\right)=\operatorname{Rad}(A)^{\mathrm{cl}}=A^{\mathrm{cl}} \cap \operatorname{Rad}(K A)$. 
Proof. $\operatorname{Rad}(A)$ satisfies the identity $x^{n}=0$, which can be expressed in terms of polynomial relations (cf. Proposition 3.17); therefore $\operatorname{Rad}(A)^{\mathrm{cl}}$ is also nil. But clearly $\operatorname{Rad}(A)^{\mathrm{cl}} \subseteq A^{\mathrm{cl}}$, so $\operatorname{Rad}(A)^{\mathrm{cl}} \subseteq \operatorname{Rad}\left(A^{\mathrm{cl}}\right)$.

Likewise $\operatorname{Rad}(A)^{\mathrm{cl}} \subseteq K \operatorname{Rad}(A)$, by Lemma 3.6, which in turn is a nilpotent ideal of $K A$ and thus contained in $\operatorname{Rad}(K A)$. This proves $\operatorname{Rad}(A)^{\mathrm{cl}} \subseteq A^{\mathrm{cl}} \cap \operatorname{Rad}(K A)$. But the latter is a nilpotent ideal of $A^{\mathrm{cl}}$ so is in $\operatorname{Rad}\left(A^{\mathrm{cl}}\right)$, completing the circle of inclusions.

The inclusion $K \operatorname{Rad}(A) \subseteq \operatorname{Rad}(K A)$ can in general be a proper one. In fact, when $A$ is not maximally Zariski-closed in $B$, we can have $\operatorname{Rad}(K A) \neq 0$ even if $A$ is simple.

Example 3.22. Suppose $L / F$ is an inseparable field extension of dimension $p$, viewed as an $F$-subalgebra of $\mathrm{M}_{p}(K)$, where $K$ is the algebraic closure of $F$. Then

$$
K L \cong K\left[z \mid z^{p}=0\right]
$$

has non-trivial radical.

Since $F$ is necessarily infinite, $L^{\mathrm{cl}}=K L$.

We are ready to turn to the Zariski closure of factor images.

Proposition 3.23. Suppose $A$ is Zariski-closed in $B=K A$. Then $A / \operatorname{Rad}(A)$ is Zariski-closed in $B / \operatorname{Rad}(B)$.

Proof. Let $J=\operatorname{Rad}(B)$. By Corollary 3.15, $A /(A \cap J)$ is Zariski-closed in $B / J$. But we are done, since $A \cap J=\operatorname{Rad}(A)$ by Proposition 3.21 .

Proposition 3.24. Suppose $A$ is Zariski-closed in $B$, and $z \in \operatorname{Cent}(B)$. Then $A / \mathrm{Ann}_{A} z$ is Zariski-closed in $B / \operatorname{Ann}_{B} z$.

Proof. $\mathrm{Ann}_{A} z=A \cap \mathrm{Ann}_{B} z$, so again we apply Corollary 3.15.

Zariski-closed algebras behave strikingly similarly to finite dimensional algebras over an algebraically closed field.

Proposition 3.25. If $A^{\mathrm{cl}}$ is simple, then it is a matrix algebra, either over a finite field or over the algebraically closed field $K$.

Proof. As a PI-algebra, $A^{\text {cl }}$ is finite dimensional over its center $F$, and thus $A^{\text {cl }} \cong$ $M_{t}(D)$ for some finite dimensional division algebra $D$. If $F$ is infinite, then $A$ is finite dimensional over the algebraically closed field $K$ by Proposition 3.7, and thus $A^{\text {cl }}=M_{t}(K)$. On the other hand, if $F$ is finite, then $D=F$ by Wedderburn's Theorem 2.5, so $A^{\mathrm{cl}} \cong M_{t}(F)$.

Our next goal is to obtain a Wedderburn decomposition for a Zariski-closed algebra, into radical and semisimple parts (when $F$ is finite, as the other case is trivial). When describing intrinsic ring-theoretic properties of a Zariski-closed algebra $A$, we do not refer explicitly to $B$, and thus we choose $B$ as we wish. Usually we take $B=K A$. Here is an example of this point of view.

Lemma 3.26. If $A$ is Zariski-closed in the $K$-algebra $B$, then $\operatorname{Cent}(A)$ is Zariskiclosed.

Proof. An element of $A$ is in $\operatorname{Cent}(A)$ iff it commutes with a (finite) base of $B$, so we are done by Proposition 3.17 . 
Proposition 3.27. If $A$ is prime and Zariski-closed, then $A$ is a matrix algebra (either over a finite field or over the algebraically closed field $K$ ).

Proof. We choose $B=K A$. But then $\operatorname{Cent}(A)$ is a Zariski-closed domain and must be either finite (and thus a field) or $K$ itself. Hence $\operatorname{Cent}(A)$ is a field, so $A$ is a prime PI-algebra whose center is a field, implying $A$ is simple, so we are done by Proposition 3.25.

Theorem 3.28. Suppose $A$ is semiprime and Zariski-closed. Then $A$ is semisimple, namely isomorphic to a direct product of matrix algebras over fields.

Proof. By Proposition 3.20, we may assume $A$ is maximally closed in $B=K A$. But $\operatorname{Rad}(B)$ is a nilpotent ideal, so would intersect $A$ at a nilpotent ideal, contrary to hypothesis unless $\operatorname{Rad}(B)=0$. Hence $B=S_{1} \times \cdots \times S_{t}$ is a direct product of simple $K$-algebras. By Corollary [3.15, the projection $A_{i}$ of $A$ into $S_{i}$ is Zariskiclosed. Furthermore, the $A_{i}$ are prime, since otherwise, taking non-zero ideals $I_{1}, I_{2}$ of $A_{i}$ with $I_{1} I_{2}=0$, we have $\left(I_{1} K\right)\left(I_{2} K\right)=0$ in $S_{i}$, contrary to $S_{i}$ prime. But then, by Proposition 3.27, $A_{i}$ is a matrix algebra over a field. Writing $S_{i}=B / P_{i}$ for maximal ideals $P_{i}$ of $B$, we have $A_{i} \approx A /\left(P_{i} \cap A\right)$, implying $P_{i} \cap A$ are maximal ideals of $A$, with $\bigcap_{i=1}^{t}\left(P_{i} \cap A\right)=0$. Hence $A$ is semisimple.

Corollary 3.29. If $A$ is Zariski-closed, then $\operatorname{Rad}(A)$ is also the Jacobson radical of $A$.

Proof. $A / \operatorname{Rad}(A)$ is semiprime, and thus semisimple, implying $\operatorname{Rad}(A)$ is also the Jacobson radical.

Let us recall some technical ring-theoretic results from $\mathrm{R} 2$. Any nil ideal is idempotent-lifting, by [R2, Corollary 1.1.28]. An algebra $A$ is semiperfect when $\operatorname{Rad}(A)$ is nil and $A / \operatorname{Rad}(A)$ is semisimple, so we instantly have the following result:

Corollary 3.30. Any Zariski-closed algebra $A$ is semiperfect.

Proposition 3.31. If $A$ is Zariski-closed, then so is $A / \operatorname{Rad}(A)$.

Proof. We may assume $B=K A$ (Remark 3.8(2)) and then apply Proposition 3.23.

We can now find an analog to the Krull-Schmidt theorem.

Theorem 3.32. If $A$ is Zariski-closed, then there is a direct sum decomposition $A=\bigoplus_{i=1}^{t} A e_{i}$ of $A$ into indecomposable modules, and this decomposition is unique up to isomorphism and permutation of components.

Proof. By [R2, Lemma 2.7.18], since $A$ is semiperfect.

Our main structural result is an analog of Wedderburn's Principal Theorem, Theorem 2.1, which played such a crucial role in Kemer's proof of Specht's conjecture in characteristic 0 . This result is the version that we need in characteristic $p$.

Theorem 3.33. If $A=A^{\mathrm{cl}}$, then $A$ has a Wedderburn decomposition $A=S \oplus J$, where $J=\operatorname{Rad}(A)$ and $S \cong A / J$ is a subalgebra of $A$.

Proof. By Wedderburn's Principal Theorem, [R2, Theorem 2.5.37(Case I)] it is enough to prove $A / J$ is split semisimple. But $A / J$ is Zariski-closed by Proposition 3.23 so we are done by Theorem 3.28 . 


\subsection{Subdirect decompositions of Zariski-closed algebras.}

Remark 3.34. Suppose $B$ is a subdirect product of $K$-algebras $B_{1}$ and $B_{2}$. Since annihilator ideals can be defined through polynomial relations, a Zariski-closed subalgebra $A$ of $B$ is a subdirect product of Zariski-closed algebras, and arguing by induction on $[B: K]$, we may conclude that any Zariski-closed algebra $A$ is a finite subdirect product of Zariski-closed algebras whose linear closures are subdirectly irreducible.

Of course, if $A$ is the subdirect product of $A_{1}, \ldots, A_{m}$, then

$$
\operatorname{id}(A)=\operatorname{id}\left(A_{1} \times \cdots \times A_{m}\right)=\bigcap_{i=1}^{m} \operatorname{id}\left(A_{i}\right),
$$

thereby reducing the study of $\operatorname{id}(A)$ to the subdirectly irreducible case.

Let us summarize what we have done so far and indicate what is still missing. Suppose $A$ is Zariski-closed with linear closure $B$. By Wedderburn's Principal Theorem, Theorem 2.1, we can write $B=S \oplus J$, where $S$ is the semisimple part and $J$ is the radical part, and we may assume that $B$ is subdirectly irreducible and has block triangular form, so $A$ involves the same non-zero components. Furthermore, $A / J$ is semisimple and thus a direct sum of central simple algebras. We can write $A$ in upper triangular form. On the other hand, we do not yet have a good description of the relations among the components. These are treated in the next section, in particular Theorem 4.10.

\section{Types of polynomial Relations of a ZaRiski-Closed Algebra}

As before, we study an $F$-algebra $A$ contained in an $n$-dimensional $K$-algebra $B$, where $F \subseteq K$ are fields. Since PI-theory deals so extensively with the $T$-ideal $\operatorname{id}(A)$ of identities of an algebra $A$, it is reasonable to expect that the ideal of polynomial relations will play an important role in our analysis of Zariski-closed algebras in $B$.

Definition 4.1. For an $F$-algebra $A$ contained in a $K$-algebra $B$ with basis $b_{1}, \ldots$, $b_{n}, \operatorname{poly}(A) \triangleleft K\left[\lambda_{1}, \ldots, \lambda_{n}\right]$ is defined as $\operatorname{poly}(A)=\{f: f(A)=0\}$.

Our next objective is to find the "best" generators of the polynomial relations. This is a major issue, taking much of the remainder of this paper.

Unlike the Zariski closure (cf. Remark 3.3), $\operatorname{poly}(A)$ does depend on the choice of a base for $B$. In fact, the general linear group $\mathrm{GL}_{n}(K)$ acts on bases of $B$ by linear transformations and on polynomials (and ideals of polynomials) by left composition. From this point of view, relations are studied up to the action of $\mathrm{GL}_{n}(K)$ on ideals of $K\left[\lambda_{1}, \ldots, \lambda_{n}\right]$, and we may simplify the relations by proper choice of the base.

In some ways, although polynomial relations generalize polynomial identities, their ideals are easier to study than $T$-ideals, since we view them in a much more manageable algebra, the commutative algebra $K\left[\lambda_{1}, \ldots, \lambda_{n}\right]$.

Remark 4.2. Some initial remarks in studying poly $(A)$ are as follows:

(1) We may assume $A$ has some element $a=\sum \alpha_{i} b_{i}$ with $\alpha_{n} \neq 0$; otherwise we have the polynomial relation $\lambda_{n}$ that we can use to eliminate all monomials which include $\alpha_{n}$. 
(2) Since $A$ is a group under addition, we know that $0 \in A$, so $f(0)=0$ for all polynomial relations $f$ of $A$. In other words, the only polynomials in $K\left[\lambda_{1}, \ldots, \lambda_{n}\right]$ that we consider are those having constant term 0 .

Remark 4.3. poly $(A)$, being an ideal of the Noetherian ring $K\left[\lambda_{1}, \ldots, \lambda_{n}\right]$, is finitely generated. Thus all the polynomial relations of $A$ are consequences of finitely many polynomial relations.

Thus, Specht's problem becomes trivial for polynomial relations. For example, the matrix algebra $M_{n}(F)$, viewed as an $n^{2}$-dimensional affine space over the field $F$, satisfies the polynomial relations $\lambda_{i}^{q}-\lambda_{i}$ iff $F$ is finite and satisfies the identity $x^{q}-x$.

4.1. Additively closed Zariski-closed sets. Next, we adapt the well-known theory of multilinearization. Since this uses the additive structure, we focus the next investigation to this case.

Remark 4.4.

(1) An additive group $A \subseteq K^{(n)}$ acts on its set of relations $\operatorname{poly}(A)$ via translation: $a: f(\lambda) \mapsto f(\lambda+a)$.

(2) If $A$ is an $F$-space, $F^{\times}$acts on poly $(A)$ via scaling: $\alpha: f(\lambda) \mapsto f(\alpha \lambda)$.

Definition 4.5. A polynomial $f\left(\lambda_{1}, \ldots, \lambda_{n}\right) \in K\left[\lambda_{1}, \ldots, \lambda_{n}\right]$ is quasi-linear (with respect to $A$ ) if

$$
f(\lambda+a)=f(\lambda)+f(a), \quad \forall a \in A .
$$

Also, $f$ is $F$-homogeneous if there is $d \in \mathbb{N}^{+}$such that, for each $\alpha \in F$,

$$
f\left(\alpha \lambda_{1}, \ldots, \alpha \lambda_{n}\right)=\alpha^{d} f\left(\lambda_{1}, \ldots, \lambda_{n}\right) .
$$

Remark 4.6.

(1) A quasi-linear polynomial (with respect to any $A$ ) necessarily has zero constant term, for $f(\lambda)=f(\lambda+0)=f(\lambda)+f(0)$.

(2) Over an infinite field, the quasi-linear polynomials are linear. However, note that $x^{p}-x$ is a non-linear but quasi-linear $\mathbb{F}_{p}$-homogeneous polynomial.

\section{Proposition 4.7.}

(1) Suppose $A$ is an additive group. The ideal of polynomial relations of $A$ is generated by quasi-linear polynomial relations.

(2) If $A$ is an $F$-vector space, the ideal of polynomial relations of $A$ is generated by quasi-linear $F$-homogeneous polynomial relations.

Proof. (1) Suppose $f \in \operatorname{poly}(A)$. Given any $a \in A$, we define the new polynomial relation $\Delta_{a} f(\lambda)=f(\lambda+a)-f(\lambda)$. This has smaller degree than $f$, and clearly is a consequence of $f$.

On the other hand, $f$ is a formal consequence of $\left\{\Delta_{a} f: a \in A\right\}$. Indeed, since $f$ has constant term 0 (by Remark 4.2), we have $f(0)=0$, and thus

$$
f(a)=f(a)-f(0)=\Delta_{a} f(0) .
$$

If $\Delta_{a} f(A)=0$ for all $a$, this implies $f(a)=0$, so that $f(\lambda)$ is a polynomial relation of $A$.

We can thus replace $f$ by finitely many $\Delta_{a} f$, in view of Remark 4.3, and repeating this process, eventually we get $\Delta_{a} f(\lambda)=0$ for all $a \in A$, i.e., $f$ is quasi-linear. 
(2) Given $\alpha_{i} \in F_{i}$, we can define $\nabla f=f\left(\alpha \lambda_{1}, \ldots, \alpha \lambda_{n}\right)-\alpha^{d} f\left(\lambda_{1}, \ldots, \lambda_{n}\right)$, where $d$ is the (total) degree of $f$. This provides a polynomial relation with fewer non-zero monomials, as is $f-\gamma \nabla f$ (for suitable $\gamma \in K$, provided $\nabla f \neq 0$ ). On the other hand, $f=\gamma \nabla f+(f-\gamma \nabla f)$, so we continue by induction, unless $\nabla f=0$. But this means that $f$ is $F$-homogeneous.

\section{Remark 4.8.}

(1) For an additive subgroup $V \subseteq K^{(n)}$ and a polynomial $f$ quasi-linear with respect to $V$, the intersection $V \cap Z(f)$ is a group, where

$$
Z(f)=\left\{c \in K^{(n)}: f(c)=0\right\}
$$

is the variety associated to $f$. Indeed, if $f(a)=f(b)=0$, then $f(a+b)=0$, and $f(-a)+f(a)=f(-a+a)=f(0)$, implying $f(-a)=0$.

(2) In particular, if $f$ is quasi-linear with respect to $K^{(n)}$, then its variety is a group.

(3) The variety of an arbitrary quasi-linear $F$-homogeneous polynomial relation $f$ is a vector space over $F$, since if $f(a)=0$, then

$$
f(\alpha a)=f\left(\alpha a_{1}, \ldots, \alpha a_{n}\right)=\alpha^{d} f\left(a_{1}, \ldots, a_{n}\right)=0 .
$$

Having reduced to quasi-linear (perhaps also $F$-homogeneous) polynomial relations, we would like to determine their form.

Definition 4.9. Suppose Char $F=p$. Let $q=|F|$, setting $q=1$ if $F$ is infinite.

(1) A polynomial relation $f \in K\left[\lambda_{1}, \ldots, \lambda_{n}\right]$ is of weak Frobenius type if $f$ has the following form:

$$
\sum_{i=1}^{n} \sum_{j \geq 1} c_{i j} \lambda_{i}^{q_{i j}}=0
$$

where $c_{i j} \in K$ and each $q_{i j}$ is a $p$-power. (Recall that our polynomial relations have constant term 0 .)

(2) The polynomial relation $f$ is a weak $F$-Frobenius type (also known as a $q$-polynomial in the literature) if, in (1), we may take each $q_{i j}$ to be a power of $q$.

Note that weak $F$-Frobenius type (resp. weak Frobenius type) reduces to the linear polynomial relation $\sum c_{i} \lambda_{i}=0$ for $F$ infinite (resp. in characteristic 0 ). In view of Remark 4.8, the next result (which strengthens Proposition 4.7) characterizes algebraic varieties that are Abelian groups.

\section{Theorem 4.10.}

(1) The ideal of polynomial relations of an additive group $A$ is generated by polynomial relations of weak Frobenius type. Specifically, any polynomial relation is a consequence of finitely many polynomial relations of weak Frobenius type.

(2) The ideal of polynomial relations of an F-vector space $A$ is generated by polynomial relations of weak F-Frobenius type. Specifically, any quasi-linear $F$-homogeneous polynomial relation is a consequence of finitely many polynomial relations of weak F-Frobenius type. 
Proof. (1) It is enough to prove the second assertion. We write a polynomial relation $f=\sum h_{d_{1}, \ldots, d_{n}}$, where $h$ is the monomial with multidegree $d_{1}, \ldots, d_{n}$, i.e., of the form $c \lambda_{1}^{d_{1}} \cdots \lambda_{n}^{d_{n}}$. Clearly $\Delta_{a} f=\sum \Delta_{a} h_{d_{1}, \ldots, d_{n}}$, so we consider a typical monomial

$$
h_{d_{1}, \ldots, d_{n}}=c \lambda_{1}^{d_{1}} \cdots \lambda_{n}^{d_{n}} .
$$

Taking $a=\sum \alpha_{i} b_{i}$ with $\alpha_{n} \neq 0$ (cf. Remark 4.2(1)), we have

$$
\Delta_{a}(h)=c\left(\lambda_{1}+\alpha_{1}\right)^{d_{1}} \cdots\left(\lambda_{n}+\alpha_{n}\right)^{d_{n}}-c \lambda_{1}^{d_{1}} \cdots \lambda_{n}^{d_{n}},
$$

so the highest monomial not cancelled (under the lexicographic order giving highest weight to $\lambda_{1}$ ) is

$$
d_{n} \alpha_{n} c \lambda_{1}^{d_{1}} \cdots \lambda_{n}^{d_{n}-1} .
$$

But this must be 0 , so $d_{n} \alpha_{n} c$ must be 0 in $K$, i.e. $p \mid d_{n}$, where $p=\operatorname{Char}(K)$. Continuing in this vein, we see that the highest term in $\Delta_{a}(h)$ is

$$
\alpha_{n}^{q} c\left(\begin{array}{c}
d_{n} \\
q
\end{array}\right) \lambda_{1}^{d_{1}} \cdots \lambda_{n}^{d_{n}-q}
$$

for some $p$-power $q$. This is a contradiction unless it is cancelled by $\Delta_{a}\left(h^{\prime}\right)$ for some other monomial

$$
h^{\prime}=c^{\prime} \lambda_{1}^{d_{1}^{\prime}} \cdots \lambda_{n}^{d_{n}^{\prime}} .
$$

By maximality assumption on the degrees, we must have $d_{i}^{\prime}=d_{i}$ for all $i \leq n-1$, and $d_{n}^{\prime}=d+n+q^{\prime}$ for some $p$-power $q^{\prime}$. Then $\Delta_{a}\left(h^{\prime}\right)$ contains the term

$$
\alpha_{n}^{q^{\prime}} c^{\prime}\left(\begin{array}{c}
d_{n} \\
q^{\prime}
\end{array}\right) \lambda_{1}^{d_{1}} \cdots \lambda_{n}^{d_{n}-q^{\prime}}
$$

Perhaps other terms of this form come from other monomials, but the upshot is that there is a linear combination of $p$-powers of $a_{n}$ that are 0 . But this is true for each $a_{n}$, and thus yields a polynomial relation $g\left(\lambda_{n}\right)$. Applying $\Delta_{\alpha}$ to $g\left(\lambda_{n}\right)$ for every $\alpha \in K$ enables us to reduce the power, unless $\Delta_{\alpha}\left(g\left(\lambda_{n}\right)\right)=0$ for all $\alpha$, i.e., $g$ is quasi-linear. But in this case we can add $g$ to our list of polynomial relations and use $g$ to reduce the degree of $f$ in $\lambda_{n}$.

Thus one continues until $\lambda_{n}$ does not appear in the highest monomial of $f$. Applying the same argument whenever a monomial has at least two indeterminates in it, we eventually reach the situation in which each monomial has a single indeterminate, i.e., $h_{i}=\sum_{i} c_{i j} \lambda_{i}^{d_{i j}}$. Applying $\Delta$ lowers the degree unless every $d_{i j}$ is a $p$-power, as desired.

(2) Continuing (1), applying $\nabla$ (as defined in the proof of Proposition 4.742) , lowers the degree unless every $d_{i j}$ is a $q$-power, as desired.

The claim of Example 2.7(2) follows as an immediate consequence, for if the algebra satisfied any extra identity its corresponding polynomial relations must come from the fact that $\sigma$ was Frobenius.

4.2. Multiplicatively closed Zariski-closed sets. Our next theorem is not needed for our exposition, since we never deal with multiplicatively closed subvarieties of $K^{(n)}$ unless they are algebras. Nevertheless, the result is interesting in its own right and complements the other results.

Example 4.11. The subvariety $(K \times\{0\}) \cup(\{0\} \times K)$ of $K^{(2)}$ is defined by the polynomial relation $\lambda_{1} \lambda_{2}$. 
Theorem 4.12. Suppose $A$ is a Zariski-closed (multiplicative) submonoid of $K^{(n)}$. Then the polynomial relations of $A$ are generated by polynomial relations of the form

$$
\lambda_{i_{1}} \cdots \lambda_{i_{t}}=0, \quad \lambda_{1}^{i_{1}} \cdots \lambda_{n}^{i_{n}}=\lambda_{1}^{j_{1}} \cdots \lambda_{n}^{j_{n}} \quad \text { for } \quad i_{1}, \ldots, i_{n}, j_{1}, \ldots, j_{n} \in \mathbb{Z} .
$$

Proof. To simplify notation, we write $\mathbf{i}$ for $i_{1}, \ldots, i_{n}, \lambda^{\mathbf{i}}$ for $\lambda_{1}^{i_{1}} \cdots \lambda_{n}^{i_{n}}$, and $\alpha^{\mathbf{i}}$ for $\alpha_{1}^{i_{1}} \cdots \alpha_{n}^{i_{n}}$. On the other hand, $\alpha^{m} \lambda^{\mathbf{i}}$ designates $\alpha_{1}^{m} \lambda_{1}^{i_{1}} \cdots \alpha_{n}^{m} \lambda_{n}^{i_{n}}$.

Take any polynomial relation $f=\sum c_{\mathbf{i}} \lambda_{1}^{i_{1}} \cdots \lambda_{n}^{i_{n}}=\sum_{\mathbf{i}} c_{\mathbf{i}} \lambda^{\mathbf{i}}$. By definition, $f(\alpha)=0$ for any $\alpha \in A$, and thus $f\left(\alpha^{j}\right)=0$ for each $j$, since $A$ is assumed multiplicative.

Cancelling out any $\lambda_{i}$ appearing in a polynomial relation $\lambda_{i}=0$, we induct on the number of indeterminates in $f$ and then on the number of monomials of $f$. Take any point $\left(\alpha_{1}, \ldots, \alpha_{n}\right)$. For $\gamma \in F$, we write $f_{\gamma}$ for the sum of those monomials $c_{\mathbf{i}} \lambda^{\mathbf{i}}$ for which $\alpha^{\mathrm{i}}=\gamma$. Then by definition, $f=\sum f_{\gamma}$. But

$$
f\left(\alpha^{m} \lambda\right)=\sum c_{i} \alpha_{1}^{m i_{1}} \cdots \alpha_{n}^{m i_{n}} \lambda_{1}^{i_{1}} \cdots \lambda_{n}^{i_{n}}=\sum \gamma^{m} f_{\gamma}(\lambda)
$$

so by a Vandermonde argument, we see that each $f_{\gamma}$ is a polynomial relation of $A$.

Thus, one can separate $f$ into a sum of polynomial relations involving fewer monomials (and conclude by induction) unless (comparing monomial by monomial) all the $\alpha_{\mathbf{i}}$ are equal.

But this means, for each monomial $\lambda_{1}^{i_{1}} \cdots \lambda_{n}^{i_{n}}$ and $\lambda_{1}^{j_{1}} \cdots \lambda_{n}^{j_{n}}$, that $A$ satisfies the equalities

$$
\alpha_{1}^{i_{1}} \cdots \alpha_{n}^{i_{n}}=\alpha_{1}^{j_{1}} \cdots \alpha_{n}^{j_{n}}
$$

for all $\left(\alpha_{1}, \ldots, \alpha_{n}\right) \in A$, so that

$$
\lambda_{1}^{i_{1}} \cdots \lambda_{n}^{i_{n}}=\lambda_{1}^{j_{1}} \cdots \lambda_{n}^{j_{n}}
$$

is a polynomial relation of $A$. In other words, $\alpha=\alpha_{1}^{i_{1}} \cdots \alpha_{n}^{i_{n}}$ is independent of the choice of the monomial $\lambda_{1}^{i_{1}} \cdots \lambda_{n}^{i_{n}}$ of $f$, so $\alpha^{m} f$ is an identity. But now, working backwards,

$$
0=f\left(\alpha_{1}, \ldots, \alpha_{n}\right)=\alpha f(1, \ldots, 1),
$$

implying $\sum c_{i}=f(1, \ldots, 1)=0$. This implies that

$$
f=\sum_{i} c_{i}\left(\lambda_{1}^{i_{1}} \cdots \lambda_{n}^{i_{n}}-\lambda_{1}^{j_{1}} \cdots \lambda_{n}^{j_{n}}\right)
$$

is a consequence of the relations (3).

4.3. Polynomial relations of commutative algebras. Now let us utilize the fact that $A$ is an $F$-algebra.

Definition 4.13. A polynomial relation $f$ is of Frobenius type if it has one of the following three forms, where $p=\operatorname{Char}(F)$ :

(i) $\lambda_{i}=0$,

(ii) $\lambda_{i}-\lambda_{i}^{s}=0$, where $s$ is a $p$-power, or

(iii) $\lambda_{i}-\lambda_{j}^{s}=0, j \neq i$, where $s$ is a $p$-power.

The polynomial relation $f$ has $F$-Frobenius type if, in (ii) and (iii), $s$ is a $q$-power, $q=|F|$ (where, as usual, we put $q=1$ if $F$ is infinite). 
Lemma 4.14. Suppose $A$ is an additive subgroup of $K^{(n)}$, defined by polynomial relations of the form $\lambda_{i}=0$ and $\lambda_{i}^{q_{i}}=\lambda_{j}^{q_{j}}$ for natural numbers $q_{i}$ and $q_{j}$. Then any such relation is equivalent to a polynomial relation of Frobenius type (ii) or (iii).

Proof. First we discard all components $i$ for which $\lambda_{i}=0$ holds. Next, assuming $q_{i} \leq q_{j}$, one could then factor out $x^{q_{j}-q_{i}}$ (since $K$ is a field) to get $\lambda_{i}-\lambda_{j}^{q}$ for some $q \geq 1$. We are done if $q=1$, so assume $q>1$. This relation holds for $\lambda_{i}=\lambda_{j}=1$, so additivity of $A$ gives the relation

$$
\left(\lambda_{i}+1\right)-\left(\lambda_{j}+1\right)^{q}=\left(\lambda_{1}-\lambda_{j}^{q}\right)+\sum_{\ell=1}^{q-1}\left(\begin{array}{l}
q \\
\ell
\end{array}\right) \lambda_{j}^{\ell} .
$$

If some $\left(\begin{array}{l}q \\ \ell\end{array}\right) \neq 0$, this translates to algebraicity of the $j$ component of $A$, which must thus be defined in a finite subfield of $K$, say of dimension $m$ over $\mathbb{F}_{p}$, and, as is well known, every polynomial in $x$ is satisfied by the field or is a multiple of $x^{p^{m}}-x$, and we have reduced to type (ii).

Thus we may assume that $\left(\begin{array}{l}q \\ \ell\end{array}\right)=0$ for all $1 \leq \ell<q$. But this clearly implies $K$ has positive characteristic $p>0$, and therefore $q$ is a power of $p$ (since otherwise, if $q^{\prime}$ is the highest power of $p$ less than $q$, then $\left(\begin{array}{c}q \\ q^{\prime}\end{array}\right) \neq 0$ in $\left.K\right)$.

We may now assume $f=\lambda_{i}-\lambda_{j}^{s}$. Write $s=q^{j} t$ for $t$ prime to $q$. Then $f$ reduces to the polynomial relation, $\lambda_{i}-\lambda_{j}^{t}$. But taking $\alpha \in F$ with $\alpha^{t} \neq \alpha$, we see that

$$
\alpha^{t}\left(\lambda_{i}-\lambda_{j}^{t}\right)-\left(\alpha \lambda_{i}-\alpha^{t} \lambda_{j}^{t}\right)=\left(\alpha^{t}-\alpha \lambda_{i}\right)
$$

yielding the polynomial relation $\lambda_{i}$ and thus also $\lambda_{j}$.

Theorem 4.15. Suppose $A$ is a commutative, semiprime Zariski-closed F-subalgebra of a finite dimensional commutative $K$-algebra $B$. Then poly $(A)$ is generated by finitely many polynomial relations of Frobenius type.

Proof. We can write $A \subseteq K_{1} \times \cdots \times K_{t}$, where each $K_{i} \approx K$. By Theorem 4.10 , it is enough to consider polynomial relations of weak $F$-Frobenius type, i.e. of the form $f=\sum_{i=1}^{n} \sum_{j \geq 1} c_{i j} \lambda_{i}^{q_{i j}}$. If we have any relations of type (i), we can simply remove all terms with $\lambda_{i}$, and ignore $\lambda_{i}$.

The constant term of $f$ is 0 , where the $q_{i j}$ are powers of $p$. If all the $q_{i j}$ are divisible by $p$, then we may take the $p^{\text {th }}$ root and still have a polynomial relation, so we may assume that some monomial of $f$ is linear. For convenience we assume $f$ has a monomial linear in $\lambda_{1}$.

If $A$ satisfies a polynomial relation only involving $\lambda_{i}$, this means that the projection of $A$ onto $K_{i}$ is a finite field $F_{i}$, which, if non-trivial, satisfies some identity $g_{i}=\lambda_{i}^{q}-\lambda_{i}$, where we take $q$ minimal possible. But it is well known that every polynomial satisfied by all elements of $F_{i}$ is a consequence of $g_{i}$, so we may assume that all polynomial relations involving only $\lambda_{i}$ are a consequence of $g_{i}$.

We claim that modulo the $g_{i}$, either $f$ becomes 0 or $f$ yields some polynomial relation of Frobenius type (iii). Suppose $\lambda_{1}^{i}$ appears with two differing degrees. Write $f=\sum f_{i} \lambda^{i}$. If each $f_{i}$ is a polynomial relation, then we continue inductively. Otherwise take some non-zero value and conclude that $F_{1}$ is algebraic of bounded degree over $F$, and thus is finite, yielding a polynomial relation of type (ii), which thus is a consequence of $g_{i}$. 
Thus we may assume $\lambda_{1}$ appears in a single monomial. But this means $f$ has the form $\lambda_{1}+\sum c_{j} \lambda_{j}^{q_{j}}$, so

$$
\alpha_{1} \lambda_{1}+\alpha_{j}^{q_{j}} \sum c_{j} \lambda_{j}^{q_{j}}=\alpha_{1}\left(\lambda_{1}+\sum c_{j} \lambda_{j}^{q_{j}}\right)
$$

formally, or in other words $\alpha_{1}=\alpha_{j}^{q_{j}}$ for each $j$ appearing in $f$, as desired.

Remark 4.16. We can combine Theorems 4.10 and 4.15 for any subalgebra $A$ of $B=\mathrm{M}_{n}(K)$, as follows: Let $A^{1}=A \cup \sum F e_{i i} \subseteq B$. We define a relation on $\{1, \ldots, n\}$ by saying $i \equiv j$ if there is a non-trivial Frobenius polynomial relation involving both $i$ and $j$, and we extend it by transitivity to an equivalence relation on $I=\{1, \ldots, n\}$. If $I_{u}$ is some equivalence class, then $A^{1}$ contains some element

$$
e_{u}=\sum_{i \in I_{u}} \alpha_{i} e_{i i}
$$

But then, for any $a \in A$ and any $u, v \in I$, clearly $e_{u} a e_{v} \in A^{1}$, and the only indices appearing non-trivially are from $I_{u} \times I_{v}$. We call a quasi-Frobenius polynomial relation basic if it only involves coefficients from $I_{u} \times I_{v}$ for suitable equivalence classes of $I$. In this way, we see that any quasi-Frobenius polynomial relation reduces to the sum of basic quasi-Frobenius polynomial relations.

\section{Explicit REPRESENTATIONS OF ZARISKI-CLOSED ALGEBRAS}

We have seen in Theorem 3.28 that any semiprime Zariski-closed algebra is a direct sum of matrix components and thus has a very easy representation inside $\mathrm{M}_{n}(K)$ along diagonal matrix blocks. In order to describe the structure of a Zariskiclosed algebra $A$ with non-zero nilradical $J$, we consider a faithful representation of $A$ in a matrix algebra $\mathrm{M}_{n}(K)$. Throughout, we use this particular representation to view $A \subseteq \mathrm{M}_{n}(K)$. Our object is to find a "canonical" set of matrix units of $\mathrm{M}_{n}(K)$ with which to view a Zariski-closed algebra $A$. The underlying idea, introduced by Lewin $[\mathrm{L}$ for PIs and utilized to great effect in characteristic 0 by Giambruno and Zaicev [GZ, is to write the algebra in something like upper triangular form, in order to understand the placement of radical substitutions in polynomial identities.

Example 5.1. $A=\left(\begin{array}{ccc}K & K & 0 \\ 0 & K & 0 \\ 0 & K & K\end{array}\right)$. Here $A / J$ can be identified with $\left(\begin{array}{ccc}K & 0 & 0 \\ 0 & K & 0 \\ 0 & 0 & K\end{array}\right)$, and $J$ with $\left(\begin{array}{ccc}0 & K & 0 \\ 0 & 0 & 0 \\ 0 & K & 0\end{array}\right)$. We can put $A$ into upper triangular form by switching the second and third rows and columns to get $\left(\begin{array}{ccc}K & 0 & K \\ 0 & K & K \\ 0 & 0 & K\end{array}\right)$.

Unfortunately, we may not be able to straighten out $A$ so easily, even in characteristic 0 .

Example 5.2. Let $A=\left\{\left(\begin{array}{ll}a & b \\ 0 & a\end{array}\right): a, b \in K\right\}$. This can also be viewed as the $2 \times 2$ matrix representation of the commutative algebra of dual numbers of $K$; i.e., $\left(\begin{array}{ll}a & b \\ 0 & a\end{array}\right)$ is identified with $a+b \delta$, where $\delta^{2}=0$. 
In order to represent this as a triangular matrix ring, we must identify certain components. Our objective in this section is to describe how the identifications work for a particular representation of a Zariski-closed algebra. Let us start with an easy example that may lower our expectations.

Example 5.3. $F=\mathbb{F}_{2}(\mu)$, where $\mu$ is an indeterminate over the field $\mathbb{F}_{2}$ of two elements and $K$ is its algebraic closure. Then $F$ can be represented in $K \times K$ by $a \mapsto\left(a^{2}, a^{4}\right)$, so the identification among components is the relation $\lambda_{2}=\lambda_{1}^{2}$.

A direct identification between two components, via a polynomial relation, is called gluing. When components are not glued, we say they are separated. In this paper, gluing is considered mostly along the diagonal blocks, since off-diagonal gluing turns out to be more complicated. As the above example shows, gluing need not be "onto", when taken over infinite fields.

Definition 5.4. Suppose $A$ is a Zariski-closed subalgebra of $\mathrm{M}_{n}(K)$ with radical $J$, such that $A / J=A_{1} \times \cdots \times A_{k}$ with $A_{u} \cong \mathrm{M}_{n_{u}}\left(F_{u}\right)$ for subfields $F_{u} \subseteq K$ $(u=1, \ldots, k)$.

We say $A$ is in Wedderburn block form if $n=\sum t(u) n_{u}$, and for each $u$ there are $t(u)$ distinct matrix blocks $A_{u}^{(1)}, \ldots, A_{u}^{(t(u))}$ of size $n_{u} \times n_{u}$ along the diagonal, each isomorphic to $A_{u}$, such that the given representation $\varphi: A \rightarrow \mathrm{M}_{n}(K)$ restricts to an embedding $\varphi_{u}: A_{u} \rightarrow A_{u}^{(1)} \times \cdots \times A_{u}^{(t(u))}$, where the projection $\varphi_{u}^{(\ell)}: A_{u} \rightarrow$ $A_{u}^{(\ell)}$ is an isomorphism for each $1 \leq \ell \leq t(u)$. Furthermore, $J$ is embedded into strictly upper triangular blocks (above the diagonal blocks). For each $u$, the blocks $A_{u}^{(1)}, \ldots, A_{u}^{(t(u))}$ are glued and belong to the same gluing component. For further reference, we define $m=\sum t(u)$, the total number of diagonal blocks (before gluing) in the representation of $A$.

For algebras with 1 , where 1 is represented as the identity matrix, obviously each diagonal Wedderburn block is non-empty. However, for algebras without 1, one could have some $B_{u}$ consisting only of 0 matrices. In this case we say the block $B_{u}$ is empty.

Note that the glued blocks do not have to occur consecutively; for example the semisimple part could be embedded in

$$
\left(\begin{array}{ccccc}
A_{1}^{(1)} & 0 & 0 & 0 & 0 \\
0 & A_{2}^{(1)} & 0 & 0 & 0 \\
0 & 0 & A_{1}^{(2)} & 0 & 0 \\
0 & 0 & 0 & A_{1}^{(3)} & 0 \\
0 & 0 & 0 & 0 & A_{2}^{(2)}
\end{array}\right) .
$$

The radical belongs to blocks above the diagonal; for example,

$$
\left(\begin{array}{ccccc}
A_{1}^{(1)} & 0 & 0 & J & J \\
0 & A_{2}^{(1)} & J & 0 & J \\
0 & 0 & A_{1}^{(2)} & 0 & 0 \\
0 & 0 & 0 & A_{1}^{(3)} & 0 \\
0 & 0 & 0 & 0 & A_{2}^{(2)}
\end{array}\right) .
$$


Example 5.5. The following basic illustration of Wedderburn decomposition, without gluing, appears as the "minimal algebra" in Giambruno and Zaicev [GZ, Chapter 8], which they realize as an upper block-triangular algebra

$$
\left(\begin{array}{cccc}
M_{n_{1}}(F) & * & * & * \\
0 & M_{n_{2}}(F) & * & * \\
0 & 0 & \ddots & * \\
0 & 0 & 0 & M_{n_{t}}(F)
\end{array}\right) .
$$

The Giambruno-Zaicev algebra could be thought of as the algebra-theoretic ana$\log$ of the Borel subgroups of $\mathrm{GL}(n, F)$. The semisimple part $S$ is the direct sum $\bigoplus M_{n_{i}}(F)$ of the diagonal blocks, and the radical is the part above these blocks, designated above as $(*)$.

This kind of algebra first came up in a theorem of Lewin [L], who showed that any PI-algebra $A$ with ideals $I_{1}, I_{2}$ satisfying $I_{1} I_{2}=0$ can be embedded into an algebra of the form $\left(\begin{array}{cc}A / I_{1} & * \\ 0 & A / I_{2}\end{array}\right)$.

Giambruno and Zaicev proved, in characteristic 0 , that for any variety $\mathcal{V}$ of PIalgebras, its exponent $d$ (a concept defined in terms of the asymptotics of the codimensions of $\mathcal{V}$ ) is an integer and can be realized in terms of one of these Giambruno-Zaicev algebras, as $d=\sum_{u=1}^{k} n_{u}^{2}$.

Remark 5.6. Belov B2 proved a parallel result to Giambruno-Zaicev's theorem, for Gel'fand-Kirillov dimension in any characteristic. Namely, the GK-dimension of the "generic" upper block-triangular algebra generated by $m$ generic elements is

$$
k+(m-1) \sum_{u=1}^{k} n_{u}^{2}
$$

In the same paper, Belov proved the following result: Suppose $A=S \oplus J$ is the Wedderburn decomposition of an f.d. algebra $A$, with $S=A_{1} \oplus \cdots \oplus A_{k}$ (where $A_{u}$ are the simple components). If there exist $x_{u} \in J$ such that $A_{1} x_{1} A_{2} x_{2} \cdots x_{m} A_{m} \neq$ 0 for some $m \leq k$, then $A$ contains a subalgebra isomorphic to the GiambrunoZaicev algebra built up from from $A_{1}, \ldots, A_{k}$.

Wedderburn block form refines Wedderburn's principal theorem. Indeed, it is apparent by inspection that the part of $A$ along the diagonal blocks is the semisimple part of $A$, and the part on the blocks above the diagonal is the radical part. Note that this example has not described the identifications among the $A_{u}^{(\ell)}$. Clearly there must be gluing whenever some $t(u)>1$, since $\operatorname{dim}\left(A_{u}^{(1)} \times \cdots \times A_{u}^{(t(u))}\right)=$ $t(u) \operatorname{dim} A_{u}$.

We can tighten these observations with some care. We start by assuming that $A$ is a $K$-algebra ( $K$ is algebraically closed, as always). Then each $F_{u}=K$. An easy application of an argument of Jacobson (spelled out in [R3, Theorem 25C.18]) yields:

Theorem 5.7. For $K$ algebraically closed, any finite dimensional $K$-algebra $A$ can be put into Wedderburn block form.

Corollary 5.8. Any Zariski-closed F-subalgebra $A \subseteq \mathrm{M}_{n}(K)$ can be put into Wedderburn block form. 
Proof. We put $K A$ into Wedderburn block form and then intersect down to $A$. Explicitly, $A / J$ is Zariski-closed in the semisimple part $S$ of $K A$, and $J$ is the intersection of $A$ with the part of $A K$ above the diagonal components.

5.1. Gluing Wedderburn blocks. Let us investigate gluing in the Wedderburn block form. We start with the semisimple part $\bigoplus A_{u}^{(\ell)}$ (of the blocks along the diagonal). By definition, the only gluing occurs among the $A_{u}^{(\ell)}$ for the same $u$.

Remark 5.9. Suppose $\varphi_{u}^{\ell}: A_{u} \rightarrow A_{u}^{(\ell)}$ is the representation as above. Then for any $\ell, \ell^{\prime}$ in $\{1, \ldots, t(u)\}$ we have the isomorphism

$$
\varphi_{u}^{\ell, \ell^{\prime}}=\left(\varphi_{u}^{\ell}\right)^{-1} \varphi_{u}^{\ell^{\prime}}: A_{u}^{(\ell)} \rightarrow A_{u}^{\left(\ell^{\prime}\right)} .
$$

Remark 5.10. Let $1_{u}^{(\ell)}$ denote the unit element of $A_{u}^{(\ell)}$. ( $1_{u}^{(\ell)}$ is then an idempotent of $\mathrm{M}_{n}(K)$, but we want to emphasize its role in $A_{u}^{(\ell)}$.) We want to understand the isomorphisms $\varphi_{u}^{\ell, \ell^{\prime}}$ in terms of their action on the center of the block. First of all, by Lemma 3.26 since $A$ is Zariski-closed, so is $\operatorname{Cent}(A)$, which contains $\sum_{u} F_{u} \sum_{\ell} 1_{u}^{(\ell)}$. It follows (by Theorem 4.15) that all identifications in the center come from polynomial relations of Frobenius type, between pairs $e_{\ell}$ and $e_{\ell}^{\prime}$ (as $\ell, \ell^{\prime}$ run between 1 and $t(u))$; i.e., of the form

$$
\lambda_{u}^{(\ell)}{ }_{i i}-\left(\lambda_{u}^{\left(\ell^{\prime}\right)}{ }_{i i}\right)^{q},
$$

where $s$ is a power of $|F|$ (same $q$ for each $i=1, \ldots, n_{u}$ ). Here, and henceforth, $\lambda_{u}^{(\ell)}{ }_{i j}$ is the variable corresponding to the $(i, j)^{\text {th }}$ entry in the block matrix $A_{u}^{(\ell)}$. This clearly is an instance of gluing.

Since taking the power $q$ is not necessarily onto for $K$ infinite, Remark 5.10 is not symmetric, in the sense that reversing direction from $\ell^{\prime}$ to $\ell$ involves taking $q$ roots, which is possible in the variety but not over all of $K$.

In the relation above, if $\ell^{\prime}=\ell$, i.e., $\lambda_{u}^{(\ell)}{ }_{i i}=\left(\lambda_{u}^{(\ell)}{ }_{i i}\right)^{q}$ holds, then we can view $A_{u}^{(\ell)}$ as an algebra over a base field $F_{u}$ of $q$ elements.

We continue from the center of diagonal blocks to the blocks themselves.

Definition 5.11. Suppose $|F|=q$. Two diagonal blocks $A_{u}^{(\ell)}$ and $A_{u}^{\left(\ell^{\prime}\right)}$ of $A$ in $\mathrm{M}_{n}(K)$ have Frobenius gluing of exponent $d$ if there are $n_{u} \times n_{u}$ matrix units of $\mathrm{M}_{n}(K)$ such that, for large enough $\kappa$, writing $e_{i j}^{(\ell)}$ for the corresponding $n_{u} \times n_{u}$ matrix units in $A_{u}^{(\ell)}$, the isomorphism $\phi_{u}^{\ell, \ell^{\prime}}$ identifies $\sum \alpha_{i j}^{q^{d+\kappa}} e_{i j}^{(\ell)}\left(\right.$ in $A_{u}^{(\ell)}$ ) with $\sum \alpha_{i j}^{q^{\kappa}} e_{i j}^{\left(\ell^{\prime}\right)}\left(\right.$ in $\left.A_{u}^{\left(\ell^{\prime}\right)}\right)$.

This definition can be extended to gluing an arbitrary number $t$ of blocks.

In this definition, since we may take $q$-roots, $\kappa$ can be chosen to be $\max \{0,-d\}$. We could have $d=0$, in which case we call this identical gluing. The same considerations hold for an arbitrary number $t$ of glued blocks; the smallest exponent may always be assumed to be 0 . Soon we shall see that the only possible gluing on diagonal blocks is Frobenius.

Let us consider the general situation. Since Zariski-closed algebras are defined in terms of polynomial relations on the algebraically closed field $K$ and all gluing is via a homomorphism from $K$ to itself, the gluing must come from a homomorphism defined by a polynomial. 
Remark 5.12. Gluing is possible only between matrix blocks of the same size, whose centers have the same cardinality.

When $\operatorname{Char}(F)=0$, the only polynomial homomorphism is the identity, so every gluing is identical.

Proposition 5.13. If $F$ is an infinite field, any variety of $F$-algebras contains a Zariski-closed algebra whose gluing is identical.

Proof. The linear closure $A K$ is in the same variety, by Proposition 3.7 and Lemma 3.18 .

When $F$ is a finite field, we must also contend with the Frobenius endomorphism, as illustrated in Example 5.3, which we note is preserved when we pass to the Zariski closure.

The point of Definition 5.11 is that all corresponding entries in these blocks are glued in exactly the same way (although not necessarily by the identity map). This is one way in which the theory is considerably richer in characteristic $p$ than in characteristic 0 .

Theorem 5.14. Suppose $A \subseteq \mathrm{M}_{n}(K)$ is a Zariski-closed algebra, with

$$
A / J=A_{1} \times \cdots \times A_{k},
$$

a direct product of $k$ simple components. Then we can choose the matrix units of $\mathrm{M}_{n}(K)$ in such a way that $A$ has Wedderburn block form, and all identifications among the diagonal blocks are Frobenius gluing.

Proof. Fix $u=1, \ldots, k$. Fixing a set of $n_{u} \times n_{u}$ matrix units $\left\{e_{i j}: 1 \leq i, j \leq n_{u}\right\}$ of $A_{u}^{(1)}$, we then have the corresponding set of $n_{u} \times n_{u}$ matrix units $\left\{\varphi_{u}^{1, \ell^{\prime}}\left(e_{i j}\right)\right.$ : $\left.1 \leq i, j \leq n_{u}\right\}$ of $A_{u}^{(\ell)}$. We do this for each $u$, and by [R2, Proposition 1.1.25] all of these matrix units can be combined and extended to a set of matrix units for $\mathrm{M}_{n}(K)$.

Now any matrix $\sum_{i, j=1}^{n_{u}} \alpha_{i j} e_{i j}^{(\ell)}$ of $A_{u}^{(\ell)}$ is glued (via $\varphi_{u}^{\ell, \ell^{\prime}}$ ) to

$$
\sum_{i, j=1}^{n_{u}} \varphi_{u}^{\ell, \ell^{\prime}}\left(\alpha_{i j} 1_{u}\right)^{(\ell)} e_{i j}^{\left(\ell^{\prime}\right)} \in A_{u}^{\left(\ell^{\prime}\right)} .
$$

But, by Remark [5.10, there is some $q=q\left(\ell, \ell^{\prime}\right)$ such that

$$
\varphi_{u}^{\ell, \ell^{\prime}}\left(\alpha \sum_{i=1}^{n_{u}} e_{i i}^{(\ell)}\right)=\varphi_{u}^{\ell, \ell^{\prime}}\left(\alpha 1_{u}^{(\ell)}\right)=\alpha^{q} 1_{u}^{\left(\ell^{\prime}\right)}
$$

(or visa versa, as noted above). Hence $\sum_{i, j=1}^{n_{u}} \alpha_{i j} e_{i j}^{(\ell)}$ is glued to $\sum_{i, j=1}^{n_{u}} \alpha_{i j}^{q} e_{i j}^{\left(\ell^{\prime}\right)}$, as desired.

5.2. Standard notation for Wedderburn blocks. We now change the point of view somewhat, and we write each diagonal Wedderburn block as $B_{1}, \ldots, B_{m}$ in the order in which they appear on the diagonal. Thus $m=\sum_{u=1}^{k} t(u)$, where $t(u)$ is the number of blocks in the $u^{\text {th }}$ glued component. Likewise for $r<s$ we define the block $B_{r s}=B_{r} A B_{s}$. Any $B_{r s}$ can be viewed as a matrix block; in particular $B_{r r}=B_{r}$. From this point of view, $B_{r s}$ is a $B_{r}, B_{s}$-bimodule. Each $B_{r}$ 
is a subalgebra of $\mathrm{M}_{n}(K)$, although in general it is not contained in $A$. Letting $B=\sum_{r \leq s} B_{r s}$, we have the following inclusions:

$$
A \subseteq K A \subseteq B \subseteq \mathrm{M}_{n}(K) .
$$

Example 5.15. When $F$ is finite, $A=\left\{\left(\begin{array}{cc}\alpha & b \\ 0 & \alpha\end{array}\right): \alpha \in F, b \in K\right\}$ is in Wedderburn block form. Then

$$
K A=\left\{\left(\begin{array}{cc}
a & b \\
0 & a
\end{array}\right): a, b \in K\right\} \quad \text { and } \quad B=\left\{\left(\begin{array}{cc}
a & b \\
0 & a^{\prime}
\end{array}\right): a, a^{\prime}, b \in K\right\},
$$

so the inclusions $A \subset K A \subset B \subset \mathrm{M}_{2}(K)$ are all strict.

Let $T_{1} \cup \cdots \cup T_{k}$ be the gluing partition of $\{1, \ldots, m\}$; namely $r \in T_{u}$ if, in the notation of Definition 5.4, $B_{r}=A_{u}^{(\ell)}$ for some $\ell=1, \ldots, t(u)$. Thus the $u^{\text {th }}$ component of $A / J$ embeds as $\varphi_{u}: A_{u} \rightarrow \bigoplus_{r \in T_{u}} B_{r}$. We let $\tau:\{1, \ldots, m\} \rightarrow\{1, \ldots, u\}$ denote the quotient map, associating to every index $r$ the gluing class $u$ of the block $B_{r}$; thus $r \in T_{\tau(r)}$.

As always, $A$ is an algebra over a field $F$ of order $q$, a prime power. (We also permit $F$ to be infinite, although this case is easier.) We write $F_{u}$ for the field of scalar matrices of $B_{r r}$, where $u=\tau(r)$. When finite, $\left|F_{u}\right|=q^{d_{u}}$ for some number $d_{r}$.

Remark 5.16. Suppose $B_{r r}$ and $B_{s s}$ are glued blocks, with center of order $q^{d_{u}}$ for $u=\tau(r)=\tau(s)$. If $B_{r r}$ and $B_{s s}$ are glued via the Frobenius endomorphism $a \mapsto a^{q^{d}}$, note that $d$ is only well defined modulo $d_{u}$.

5.3. Sub-Peirce decomposition and the linear closure. Given a Zariski-closed algebra $A$ over $F$, represented in $\mathrm{M}_{n}(K)$, for $K$ infinite, we have the primitive idempotents $\hat{e}_{u}$ of $A(u=1, \ldots, k)$, which give rise to the Peirce decomposition $A=\bigoplus \hat{e}_{u} A \hat{e}_{v}$.

Each idempotent decomposes as a sum $\hat{e}_{u}=\sum_{r \in T_{u}} e_{r}$ of idempotents of $\mathrm{M}_{n}(K)$, where $T_{u}$ are defined in the previous subsection and we have the Peirce decomposition $B=\bigoplus e_{r} A e_{s}$. This is a fine decomposition, and in general, $e_{r} A e_{s}$ is not contained in $A$. Nevertheless, we do have the following observation.

Remark 5.17. Suppose $A=\prod_{i=1}^{m} F_{i}$ is a commutative semisimple algebra and $a=\left(\alpha_{i}\right) \in A$ is written as $a=\sum a_{j}$, where each $a_{j}$ is the sum of those Frobenius components of $a$ that are glued. Then each $a_{j} \in A^{\mathrm{cl}}$.

Definition 5.18. A primitive idempotent $\hat{e}$ of $A$ is of finite (resp. infinite) type if the base centers $F_{u}$ of the corresponding glued blocks $e_{r} \mathrm{M}_{n}(K) e_{r}$ are finite (resp. infinite).

Note that by Proposition 3.7, $F_{u} \cong K$ for any idempotent of infinite type, although there may fail to be a natural action of $K$ on the $F_{u}$ because of nonidentity gluing.

Example 5.19. (1) The primitive idempotents of

$$
A=\left\{\left(\begin{array}{ccc}
\alpha & 0 & x \\
0 & \beta & y \\
0 & 0 & \alpha^{q}
\end{array}\right): \alpha, \beta, x, y \in K\right\}
$$


are $e_{11}+e_{33}$ and $e_{22}$. Numerating the blocks into gluing components by setting $T_{1}=\{1,3\}$ and $T_{2}=\{2\}$, we have that $F_{1}, F_{2} \cong K$; however, scalar multiplication by $K$ does not preserve $F_{2}$. Indeed, $A$ is not a $K$-algebra: $\operatorname{dim}(A)=4$, while $\operatorname{dim}(K A)=5$.

(2) Let $A=\left\{\left(\begin{array}{cccc}\alpha & x & y & \lambda x \\ 0 & \beta & z & 0 \\ 0 & 0 & \alpha & 0 \\ 0 & 0 & 0 & \beta\end{array}\right): \alpha, \beta, x, y, z \in K\right\}$, where $\lambda \in K$ is fixed. The glued blocks are $T_{1}=\{1,3\}$ and $T_{2}=\{2,4\}$. Accordingly, $A=$ $A_{11} \oplus A_{12} \oplus A_{21} \oplus A_{22}$, where $A_{11}=K\left(e_{11}+e_{33}\right)+K e_{13}, A_{12}=K\left(e_{12}+\lambda e_{14}\right)$, $A_{21}=K e_{23}$ and $A_{22}=K\left(e_{22}+e_{44}\right)+K e_{24}$.

We would like to refine this description by comparing the Wedderburn decompositions of $A$ and its linear closure $K A$ (which may have more primitive idempotents), even though $A$ and $K A$ need not be PI-equivalent.

Break every gluing class $T_{u}(u=1, \ldots, k)$ into a disjoint union $T_{u}=T_{u}^{(1)} \cup \cdots \cup$ $T_{u}^{\left(c_{u}\right)}$, where blocks $B_{r}, B_{s}$ are in the same component $T_{u}^{(\mu)}$ if and only if they are glued by an identical gluing. For example, in Example 5.19(1) the decomposition is $T_{1}=\{1\} \cup\{3\}$. The idempotents $\hat{e}_{u}$ decompose, accordingly, as

$$
\hat{e}_{u}=\sum_{\mu=1}^{c_{u}} \bar{e}_{u}^{(\mu)}
$$

where $\bar{e}_{u}^{(\mu)}=\sum_{r \in T_{u}^{(\mu)}} e_{r}$. Although $\bar{e}_{u}^{(\mu)}$ are not in $A$, these elements do belong to $K A$ (since $K$ is infinite, allowing for a Vandermonde argument). Therefore, we define:

Definition 5.20. The sub-Peirce decomposition of $A$ is the restriction to $A$ of the Peirce decomposition of $K A$; cf. equation (11). Namely,

$$
A \subseteq \bigoplus A_{u v}^{\left(\mu \mu^{\prime}\right)}, \quad A_{u v}^{\left(\mu \mu^{\prime}\right)}=\bar{e}_{u}^{(\mu)} A \bar{e}_{v}^{\left(\mu^{\prime}\right)},
$$

where the sum ranges over $u, v=1, \ldots, k, \mu=1, \ldots, c_{u}$ and $\mu^{\prime}=1, \ldots, c_{v}$. We stress once more that this is not a decomposition of $A$, as the $A_{u v}^{\left(\mu \mu^{\prime}\right)}$ are contained in $K A$, but not in $A$ in general.

Notice that if all the gluing in $A$ are via the identity map, in particular (by Proposition 5.13), if $A$ is a $K$-algebra, then the sub-Peirce decomposition is identical to the Peirce decomposition.

Example 5.21. Let $A=\left\{\left(\begin{array}{cccc}\alpha & x & y & z \\ 0 & \alpha^{q} & x^{\prime} & y^{\prime} \\ 0 & 0 & \alpha & x^{\prime \prime} \\ 0 & 0 & 0 & \alpha^{q}\end{array}\right): \alpha, x, x^{\prime}, x^{\prime \prime}, y, y^{\prime}, z \in K\right\}$. There is one glued component, namely $T_{1}=\{1,2,3,4\}$, which decomposes with respect to identical gluing as $T_{1}=\{1,3\} \cup\{2,4\}$. The corresponding idempotent decomposition is $\hat{e}_{1}=\bar{e}_{1}^{(1)}+\bar{e}_{1}^{(2)}$, where $\hat{e}_{1}=1, \bar{e}_{1}^{(1)}=e_{11}+e_{33}$ and $\bar{e}_{1}^{(2)}=e_{22}+e_{44}$. The sub-Peirce components are $A_{11}^{(11)}=K \bar{e}_{1}^{(1)}+K e_{13}, A_{11}^{(12)}=K e_{12}+K e_{14}+K e_{34}$, $A_{11}^{(21)}=K e_{23}$ and $A_{11}^{(22)}=K \bar{e}_{1}^{(2)}+K e_{24}$ (similarly to the Peirce components in Example 5.19(2)). 
From one point of view, the linear closure erases all the subtlety introduced by the finiteness of $F$, as we see in the next observation.

Remark 5.22. Identity gluing in $A$ is preserved in $K A$; however, it may happen that $A=A^{\mathrm{cl}}$ and $A$ has only identical gluing, while $A \subset K A$ (see Example 5.15).

On the other hand, non-identity (Frobenius) gluing for $A$ is unglued in $K A$, as seen by applying a Vandermonde argument since $K$ is infinite. Thus $K A$ only has identical gluing.

Viewed in terms of the Peirce decomposition, a Peirce component $A_{u u}$ of $A$ may ramify in $K A$, and the corresponding primitive idempotent in $A$ becomes a sum of orthogonal idempotents in $K A$. Thus, the sub-Peirce decomposition of $A$ consists of identity-glued components of the Peirce decomposition of $K A$.

From the point of view of PI's, $K A$ satisfies all multilinear identities of $A$, although it may lose identities arising from Frobenius automorphisms, such as $x^{2} y-y x$ in the example $A=\left\{\left(\begin{array}{cc}a & b \\ 0 & a^{2}\end{array}\right): a, b \in \mathbb{F}_{4}\right\}$.

It turns out that non-identical gluing permits us to refine the decomposition further, and this is our next goal.

\subsection{Relative exponents.}

Definition 5.23. Let $B_{r}$ and $B_{r^{\prime}}$ be two glued blocks (whose centers thus have the same cardinality). By Theorem 5.14, we may assume the blocks are glued by Frobenius gluing of some exponent (cf. Definition 5.11), which we denote as $\exp \left(B_{r r^{\prime}}\right)$ and call the relative Frobenius exponent of $B_{r r^{\prime}}$. This is understood to be zero if $F$ is infinite. In fact, $\exp \left(B_{r r^{\prime}}\right)$ is only well defined modulo the dimension of $F_{r}=F_{r^{\prime}}$ over $F$ (where we interpret 'modulo infinity' as a mere integer).

The relative Frobenius exponents are used to define equivalence relations on vectors of glued indices, as follows.

Definition 5.24. Recall the definition of $T_{u}$ from Subsection 5.2 For every $1 \leq$ $u, v \leq k$, we let $T_{u, v}=\left\{(r, s) \in T_{u} \times T_{v}: r \leq s\right\}$, and define an equivalent relation on $T_{u, v}$ by setting $(r, s) \sim\left(r^{\prime}, s^{\prime}\right)$ iff $\exp \left(B_{r r^{\prime}}\right) \equiv \exp \left(B_{s s^{\prime}}\right)$ modulo $\operatorname{gcd}\left(d_{u}, d_{v}\right)$. (Recall that $d_{u}$ is the dimension of the center of the $u^{\text {th }}$ component over $F$.)

More generally, for every $t$-tuple $1 \leq u_{1}, \ldots, u_{t} \leq k$, we set

$$
T_{u_{1}, \ldots, u_{t}}=\left\{\left(r_{1}, \ldots, r_{t}\right) \in T_{u_{1}} \times \cdots \times T_{u_{t}}: r_{1} \leq \cdots \leq r_{t}\right\},
$$

and define an equivalence relation on $T_{u_{1}, \ldots, u_{t}}$ by $\left(r_{1}, \ldots, r_{t}\right) \sim\left(r_{1}^{\prime}, \ldots, r_{t}^{\prime}\right)$ if the values $\exp \left(B_{r_{1} r_{1}^{\prime}}\right), \ldots, \exp \left(B_{r_{t} r_{t}^{\prime}}\right)$ are all equivalent modulo $\operatorname{gcd}\left(d_{u_{1}}, \ldots, d_{u_{t}}\right)$. We call $t$ the length of $\gamma$.

Remark 5.25. (1) Relative exponents can be computed with respect to a fixed block in the gluing component, i.e. $\exp \left(B_{r r^{\prime}}\right)=\exp \left(B_{r_{0} r^{\prime}}\right)-\exp \left(B_{r_{0} r}\right)$. In particular, $\exp \left(B_{r r^{\prime}}\right)=-\exp \left(B_{r^{\prime} r}\right)$, and $\exp \left(B_{r r^{\prime \prime}}\right)=\exp \left(B_{r r^{\prime}}\right)+\exp \left(B_{r^{\prime} r^{\prime \prime}}\right)$.

(2) In a 'diagonal' set $T_{u, u}, \exp \left(B_{r r^{\prime}}\right) \equiv \exp \left(B_{s s^{\prime}}\right)$ iff $\exp \left(B_{r s}\right) \equiv \exp \left(B_{r^{\prime} s^{\prime}}\right)$, and so one can read the equivalence relation directly from the matrix of relative exponents.

(3) Likewise for any $t$, if $u_{1}=\cdots=u_{t}=u$, then the equivalence relation on $T_{u, \ldots, u}$ is given as follows: $\left(r_{1}, \ldots, r_{t}\right) \sim\left(r_{1}^{\prime}, \ldots, r_{t}^{\prime}\right)$ iff $\exp \left(B_{r_{i} r_{i+1}}\right) \equiv$ $\exp \left(B_{r_{i}^{\prime} r_{i+1}^{\prime}}\right)$ modulo $d_{u}$ for $i=1, \ldots, t-1$. 
(4) If $B_{r}$ and $B_{\bar{r}}$ are identically glued, then obviously $\exp \left(B_{r s}\right)=\exp \left(B_{\bar{r} s}\right)$ for any $s$. In particular

$$
\left(r_{1}, \ldots, r_{i-1}, r, r_{i+1}, \ldots, r_{t}\right) \sim\left(r_{1}, \ldots, r_{i-1}, \bar{r}, r_{i+1}, \ldots, r_{t}\right),
$$

whenever $r_{i-1} \leq r, \bar{r} \leq r_{i+1}$.

A word of caution: When all the Peirce idempotents in a given sub-Peirce vector $\left(u_{1}, \ldots, u_{t}\right)$ correspond to fields $F_{u_{i}}$ of the same size (such as all having infinite type), then the equivalence class of this vector is uniquely determined by the equivalence classes of the pairs $\left(u_{i}, u_{i+1}\right)$. However, this can fail when the $F_{u_{i}}$ have differing sizes (such as some finite and some infinite), because of the ambiguity arising from the differing exponents of the Frobenius automorphisms.

If $\left(r_{i}, r_{i+1}\right) \sim\left(r_{i}^{\prime}, r_{i+1}^{\prime}\right)$ for each $i=1, \ldots, t-1$, then the respective relative exponents are equivalent modulo $\operatorname{gcd}\left(d_{i}, d_{i+1}\right)$, so in particular they are all equivalent $\operatorname{modulo} \operatorname{gcd}\left(d_{1}, \ldots, d_{t}\right)$, and so $\left(r_{1}, \ldots, r_{t}\right) \sim\left(r_{1}^{\prime}, \ldots, r_{t}^{\prime}\right)$.

On the other hand, equivalence modulo $\operatorname{gcd}\left(d_{1}, \ldots, d_{t}\right)$ does not in general imply any equivalence modulo $\operatorname{gcd}\left(d_{i}, d_{i+1}\right)$, so, for example, $\left(r_{1}, r_{2}, r_{3}\right) \sim\left(r_{1}^{\prime}, r_{2}^{\prime}, r_{3}^{\prime}\right)$ does not even imply $\left(r_{1}, r_{2}\right) \sim\left(r_{1}^{\prime}, r_{2}^{\prime}\right)$.

Definition 5.26. We define the composition of equivalence classes, in the spirit of matrix units, as follows. Suppose $\gamma \subseteq T_{u_{1}, \ldots, u_{t}}$ and $\gamma^{\prime} \subseteq T_{v_{1}, \ldots, v_{t^{\prime}}}$. If $u_{t} \neq v_{1}$, let $\gamma * \gamma^{\prime}=\emptyset ;$ and if $u_{t}=v_{1}$, let

$$
\gamma * \gamma^{\prime}=\left\{\left(r_{1}, \ldots, r_{t-1}, r_{t}, s_{2}, \ldots, s_{t^{\prime}}\right):\left(r_{1}, \ldots, r_{t-1}, r_{t}\right) \in \gamma,\left(r_{t}, s_{2}, \ldots, s_{t^{\prime}}\right) \in \gamma^{\prime}\right\} .
$$

The composition $\gamma \circ \gamma^{\prime}$ is defined as the set of equivalence classes (of length $t+t^{\prime}-1$ ) of $T_{u_{1}, \ldots, u_{t}, v_{2}, \ldots, v_{t^{\prime}}}$ which are contained in $\gamma * \gamma^{\prime}$.

5.5. The relative Frobenius decomposition. Let $a \in A$ be an element in a Peirce component $\hat{e}_{u} A \hat{e}_{v}$ of $A$. Applying the matrix block component decomposition $a=\sum_{(r, s) \in T_{u, v}} a_{r s}$ (with $a_{r s} \in B_{r s}$ ) we have $a=\sum a^{\gamma}$, where

$$
a^{\gamma}=\sum_{(r, s) \in \gamma} a_{r s}
$$

and $\gamma$ ranges over the equivalence classes of $T_{u, v}$ defined in Definition 5.24. Thus, letting $A^{\gamma}=\left\{a^{\gamma}: a \in \hat{e}_{u} A \hat{e}_{v}\right\}$, we have the relative Frobenius decomposition

$$
A=\sum_{1 \leq u, v \leq k} \sum_{\gamma \subseteq T_{u, v}} A^{\gamma} .
$$

Proposition 5.27. If $a \in A^{\mathrm{cl}}$, then $a^{\gamma} \in A^{\mathrm{cl}}$ for each equivalence class $\gamma$ in every $T_{u, v}$.

Proof. We need to show that each glued component $a^{\gamma}$ is in $A^{\mathrm{cl}}$. Let $M_{r s}$ be the $F$ subspace of $\mathrm{M}_{n}(K)$ spanned by the $a_{r s} e_{r s}$. The natural vector space isomorphism $F \rightarrow M_{r s}$ can be used to transfer the algebra structure of $F$ to $M_{r s}$; i.e.,

$$
\left(\alpha a_{r s} e_{r s}\right)\left(\beta a_{r s} e_{r s}\right)=\alpha \beta a_{r s} e_{r s} .
$$

Note that each $M_{r s}$ is closed under these operations. With respect to this structure, $M=\bigoplus_{r, s} M_{r s}$ becomes a commutative $F$-algebra (defining the operations componentwise), and its subalgebra $\tilde{M}$ corresponding to $a^{\gamma}$ is semiprime Zariskiclosed, and thus has only Frobenius gluing, in view of Remark 5.12, But this implies $\tilde{M} \subseteq A^{\mathrm{cl}}$, as desired. 
We see that the relative Frobenius decomposition is finer than the Peirce decomposition, but coarser than the sub-Pierce decomposition (which, strictly speaking, is not a decomposition of $A$, since the components only belong to $K A$ ).

Corollary 5.28. Two blocks of the linear closure $K A$ are in the same component in the sub-Peirce decomposition iff they are identically glued. Thus, in the relations defining the algebra $A$, any two off-diagonal blocks $B_{r s}$ and $B_{r^{\prime} s^{\prime}}$ with $(r, s) \nsim\left(r^{\prime}, s^{\prime}\right)$ can be separated (see Remark 6.1 below).

Remark 5.29. Suppose $f$ is a relation of weak Frobenius type on $A$ whose variables involve the blocks $B_{r_{1}, s_{1}}, \ldots, B_{r_{\nu}, s_{\nu}}$. Assume $f$ cannot be concluded from relations on the same blocks, with fewer variables. Then the following facts hold:

(1) The diagonal blocks $B_{r_{1}}, \ldots, B_{r_{\nu}}$ are glued. (Indeed, suppose some $a \in A$ satisfies the weak Frobenius relation

$$
\sum_{i=1}^{n} \sum_{j \geq 1} c_{i j}\left(a_{r, s}^{i, j}\right)^{q_{r, s}}=0
$$

If some $B_{r}$ and $B_{r^{\prime}}$ are not glued, then we have a diagonal element $d \in A$ with the identity 1 in the $B_{r}$ block and 0 in the $B_{r^{\prime}}$ block. Then $d a=0$, contrary to the minimality of the quasi-linear relation defining the gluing.) Thus, applying some graph theory cuts down the number of generating polynomial relations even further.

(2) Each diagonal block $B_{r}$ is defined over a field whose order is at most the maximal $q_{r, s}$. (Multiply diagonal elements of $A$ by the elements in the given glued components of $A$, and apply a Vandermonde argument.)

Example 5.30. Let $A$ be the algebra of Example 5.21. For $u=v=1$, the relative exponents of $B_{r s}$ is the $(r, s)^{\text {th }}$ entry in the antisymmetric matrix $\left(\begin{array}{cccc}0 & 1 & 0 & 1 \\ \cdot & 0 & -1 & 0 \\ \cdot & \cdot & 0 & 1 \\ \cdot & \cdot & \cdot & 0\end{array}\right)$; see Remark 5.25(2). The equivalence relation on $T_{1,1}$ has classes $\{(1,2),(1,4),(3,4)\}$, $\{(2,3)\}$ and $\{(1,1),(1,3),(2,2),(2,4),(3,3),(4,4)\}$. In the notation of Example 5.21. the relative Frobenius decomposition is

$$
A=\left(A_{11}^{(11)}+A_{11}^{(22)}\right) \oplus A_{11}^{(12)} \oplus A_{11}^{(21)} .
$$

Example 5.31. Now take $A=\left\{\left(\begin{array}{cccc}\alpha & x & y & z \\ 0 & \beta^{q} & x^{\prime} & y^{\prime} \\ 0 & 0 & \beta & x^{\prime \prime} \\ 0 & 0 & 0 & \alpha^{q}\end{array}\right): \alpha, \beta, x, x^{\prime}, x^{\prime \prime}, y, y^{\prime}, z \in K\right\}$.

There are two glued components, $T_{1}=\{1,4\}$ and $T_{2}=\{2,3\}$. The non-diagonal relative Frobenius exponents are $\exp \left(B_{14}\right)=1$ and $\exp \left(B_{23}\right)=-1$, as in Example 5.30. The equivalence components are $T_{1,1}=\{(1,1),(4,4)\} \cup\{(1,4)\}$, $T_{1,2}=\{(1,2)\} \cup\{(1,3)\}, T_{2,1}=\{(2,4)\} \cup\{(3,4)\}$ and $T_{2,2}=\{(2,2),(3,3)\} \cup\{(2,3)\}$. For this algebra, the relative Frobenius decomposition recaptures the full sub-Peirce decomposition. 
5.6. Higher length decomposition. The same proof as in Proposition 5.27 yields the following more intricate result:

Remark 5.32. Let $t \geq 2$ and $1 \leq u_{1}, \ldots, u_{t} \leq k$. Let $a=\hat{e}_{u_{1}} a_{1} \hat{e}_{u_{2}} \cdots \hat{e}_{u_{t-1}} a_{t-1} \hat{e}_{u_{t}} \in$ $\hat{e}_{u_{1}} A \hat{e}_{u_{2}} \cdots \hat{e}_{u_{t-1}} A \hat{e}_{u_{t}}$, where $a_{1}, \ldots, a_{t-1} \in A$.

For every equivalence class $\gamma \subseteq T_{u_{1}, \ldots, u_{t}}$ (as defined in Definition 5.24) and $a$ as above, let $a^{\gamma}=\sum_{\left(r_{1}, \ldots, r_{t}\right) \in \gamma} e_{r_{1}} a_{1} e_{r_{2}} \cdots e_{r_{t-1}} a_{t-1} e_{r_{t}}$. Then $a^{\gamma} \in A$ and (clearly) $a=\sum_{\gamma \subseteq T_{u_{1}, \ldots, u_{t}}} a^{\gamma}$. Writing $a_{i}=\sum_{r s} \alpha_{r s}^{(i)} e_{r s}$, we have that

$$
a^{\gamma}=\sum_{\left(r_{1}, \ldots, r_{t}\right) \in \gamma} \alpha_{r_{1} r_{2}}^{(1)} \cdots \alpha_{r_{t-1} r_{t}}^{(t-1)} e_{r_{1} r_{t}} .
$$

Letting $A^{\gamma}=\left\{a^{\gamma}: a \in \hat{e}_{u_{1}} A \hat{e}_{u_{2}} \cdots \hat{e}_{u_{t-1}} A \hat{e}_{u_{t}}\right\}$ (where we implicitly take advantage of the fact that $\gamma$ determines $\left(u_{1}, \ldots, u_{t}\right)$ ), we have the $t$-fold relative Frobenius decomposition

$$
A=\sum_{1 \leq u_{1}, \ldots, u_{t} \leq k} \sum_{\gamma \subseteq T_{u_{1}, \ldots, u_{t}}} A^{\gamma} .
$$

While equation (6) is clearly a direct sum, this is no longer the case for $t \geq 2$ in (8), as demonstrated in Example 5.39 below.

Remark 5.33. The decomposition of Remark 5.32 becomes finer as the length of the classes increases. Indeed, for $\gamma$ of length $t+1$ and $1<i<t+1$, let $\pi_{i}$ denote the map $\{1, \ldots, m\}^{t+1} \rightarrow\{1, \ldots, m\}^{t}$, forgetting the $i^{\text {th }}$ entry. If $\gamma \subseteq T_{u_{1}, \ldots, u_{t+1}}$, then $\pi_{i}(\gamma)$ is contained in an equivalence class of $T_{u_{1}, \ldots, u_{i-1}, u_{i+1}, \ldots, u_{t+1}}$. For any equivalence class $\hat{\gamma} \subseteq T_{u_{1}, \ldots, u_{t}}$, one uses $A=\sum A \hat{e}_{v} A$ to show that

$$
A^{\hat{\gamma}}=\sum_{v=1}^{k} \sum_{\gamma} A^{\gamma},
$$

where, for each $v, \gamma$ ranges over all equivalence classes of $T_{u_{1}, \ldots, u_{i-1}, v, u_{i}, \ldots, u_{t}}$ such that $\pi_{i}(\gamma)=\hat{\gamma}$ ( $k$ is the number of gluing components).

Products of the components obtained in this manner can be easily computed via the following formula.

Remark 5.34. If $\gamma$ and $\gamma^{\prime}$ are equivalence classes of arbitrary length, then we have the 'thick filtration' formula

$$
A^{\gamma} \cdot A^{\gamma^{\prime}}=\sum_{\gamma^{\prime \prime} \in \gamma \circ \gamma^{\prime}} A^{\gamma^{\prime \prime}}
$$

(where the composition is defined in Definition 5.26). In particular, the decomposition of length $t+1$ is a refinement of the product of the basic decomposition, of length 2, with the decomposition of length $t$.

Remark 5.35. If $A \subseteq \mathrm{M}_{n}(K)$ is an $F$-algebra (not necessarily Zariski-closed), we can define $\check{A}$ to be the extension of $A$ in $K A$ generated by all sub-Pierce components of elements of $A$. Thus, $A \subseteq A^{\mathrm{cl}} \subseteq \check{A} \subseteq K A$.

For example, if $A=\left\{\left(\begin{array}{cc}\alpha^{p^{n}} & a \\ 0 & \alpha\end{array}\right): \alpha \in F_{1}, a \in K\right\}$ (cf. Example 2.7(1)), then $\check{A}=\left(\begin{array}{cc}F_{2} & K \\ 0 & F_{1}\end{array}\right)$, where $F_{2}=\left\{\alpha^{p}: \alpha \in K_{1}\right\}$. 
In particular, if $A \subseteq \mathrm{M}_{n}(K)$ is a generic algebra of a given variety, generated by generic elements (see Section 7 below), then $\check{A}$ is naturally graded by the matrix components of $\mathrm{M}_{n}(K)$, although $A$ itself is not graded.

5.7. Interaction with the radical. The decompositions in (1)-(3) above can be refined further via the decomposition $A=S \oplus J$ to the semisimple and radical parts. For example $A_{(u v)} \subseteq J$ for $u \neq v$, but $A_{(u u)}$ is a (non-unital) subalgebra of $A$, with $\operatorname{Rad}\left(A_{(u u)}\right)=J \cap A_{(u u)}$.

Remark 5.36. Let $\gamma \subseteq T_{u_{1}, \ldots, u_{t}}$ be an equivalence class, and fix $1 \leq i<t$. By definition, $A^{\gamma}=\left\{a^{\gamma}\right\}$, ranging over

$$
\begin{aligned}
a & =\hat{e}_{u_{1}} a_{1} \hat{e}_{u_{2}} \cdots \hat{e}_{u_{t-1}} a_{t-1} \hat{e}_{u_{t}} \\
& \in \hat{e}_{u_{1}} A \cdots \hat{e}_{u_{i}} A \hat{e}_{u_{i+1}} \cdots A \hat{e}_{u_{t}} \\
& =\hat{e}_{u_{1}} A \cdots \hat{e}_{u_{i}} S \hat{e}_{u_{i+1}} \cdots A \hat{e}_{u_{t}}+\hat{e}_{u_{1}} A \cdots \hat{e}_{u_{i}} J \hat{e}_{u_{i+1}} \cdots A \hat{e}_{u_{t}} .
\end{aligned}
$$

If $u_{i} \neq u_{i+1}$, then $\hat{e}_{u_{i}} S \hat{e}_{u_{i+1}}=0$, so we may assume $a_{i} \in J$. Otherwise, the computation shows that

$$
a^{\gamma}=\sum_{\left(r_{1}, \ldots, r_{t}\right) \in \gamma, r_{i}<r_{i+1}} e_{r_{1}} a_{1} e_{r_{2}} \cdots e_{r_{t-1}} a_{t-1} e_{r_{t}}
$$

when $a_{i} \in J$, while

$$
a^{\gamma}=\sum_{\left(r_{1}, \ldots, r_{t}\right) \in \gamma, r_{i}=r_{i+1}} e_{r_{1}} a_{1} e_{r_{2}} \cdots e_{r_{t-1}} a_{t-1} e_{r_{t}}
$$

when $a_{i} \in S$. In other words, we refine the decomposition (8) by separating the conditions $r_{i} \leq r_{i+1}$ on an equivalence class $\gamma=\left\{\left(r_{1}, \ldots, r_{t}\right)\right\}$ to one of the conditions $r_{i}=r_{i+1}$ or $r_{i}<r_{i+1}$.

We say the index $i$ has type 0 in $\gamma$ if $r_{i}=r_{i+1}$ for every $\left(r_{1}, \ldots, r_{t}\right) \in \gamma$ and has type 1 if $r_{i}<r_{i+1}$ for every $\left(r_{1}, \ldots, r_{t}\right) \in \gamma$. For example, if $u_{i} \neq u_{i+1}$, then $i$ has type 1. An equivalence class can be decomposed as a union $\gamma=\gamma^{(0)} \cup \gamma^{(1)}$, where $\gamma^{(0)}=\left\{\left(r_{1}, \ldots, r_{t}\right) \in \gamma: r_{i}=r_{i+1}\right\}$ and $\gamma^{(1)}=\left\{\left(r_{1}, \ldots, r_{t}\right) \in \gamma: r_{i}<r_{i+1}\right\}$. This process can be repeated for every $i$, and the resulting sub-classes are called fully refined classes.

One can multiply the components corresponding to refined classes, as described for standard components in Remark 5.34 .

If $\gamma^{*}$ is a fully refined equivalence class, let the weight $\omega\left(\gamma^{*}\right)$ denote the number of indices $i$ of type 1 in $\gamma^{*}$. In particular, any components having $\omega\left(\gamma^{*}\right)$ greater than the nilpotence index of $J$ must be zero. By construction, $A^{\gamma^{*}} \subseteq J^{\omega\left(\gamma^{*}\right)}$. Moreover, $J^{\ell}=\sum_{\operatorname{len}\left(\gamma^{*}\right)=t, \omega\left(\gamma^{*}\right) \geq \ell} A^{\gamma^{*}}$ for every $t \geq \ell$.

Lemma 5.37. Suppose $\gamma^{*} \subseteq T_{u_{1}, \ldots, u_{k}}$ is a refined equivalence class, with the index $i$ having type 0 . Let $\gamma^{\prime} \subseteq T_{u_{1}, \ldots, u_{i}, u_{i+2}, \ldots, u_{k}}$ be the equivalence class obtained by removing the $(i+1)$ th entry from each vector in $\gamma^{*}$. Then $A^{\gamma^{*}}=A^{\gamma^{\prime}}$.

Proof. It is clear that $A^{\gamma^{*}} \subseteq A^{\gamma^{\prime}}$, and if

$$
a^{\gamma^{\prime}}=\sum_{\left(r_{1}, \ldots, r_{i}, r_{i+2}, \ldots, r_{t}\right) \in \gamma^{\prime}} e_{r_{1}} a_{1} e_{r_{2}} \cdots e_{r_{i}} a_{i+1} e_{r_{i+2}} \cdots e_{r_{t-1}} a_{t-1} e_{r_{t}}
$$


for some $a_{1}, \ldots, a_{i-1}, a_{i+1}, \ldots, a_{t-1} \in A$, then, taking $a_{i}=1$,

$$
a^{\gamma^{*}}=\sum_{\left(r_{1}, \ldots, r_{i}, r_{i+1}, r_{i+2}, \ldots, r_{t}\right) \in \gamma^{*}} e_{r_{1}} a_{1} e_{r_{2}} \cdots e_{r_{i}} a_{i} e_{r_{i+1}} a_{i+1} e_{r_{i+2}} \cdots e_{r_{t-1}} a_{t-1} e_{r_{t}}
$$

is equal to $a^{\gamma^{\prime}}$, since $e_{r_{i}} a_{i} e_{r_{i+1}}=e_{r_{i}}$ for all vectors in $\gamma^{*}$.

Proposition 5.38. Every homogeneous component of a fully refined equivalence class has the form $A^{\gamma^{*}}$, where all indices in $\gamma^{*}$ have type 1 . In particular, len $\left(\gamma^{*}\right)=$ $\omega\left(\gamma^{*}\right) \leq \nu$.

Example 5.39. Consider the $F$-algebra

$$
A=\left\{\left(\begin{array}{cccccc}
a & 0 & * & * & * & * \\
0 & a & x & y & * & * \\
0 & 0 & a^{q} & x^{\prime} & * & * \\
0 & 0 & 0 & a & \alpha x & \alpha \alpha^{\prime} y \\
0 & 0 & 0 & 0 & a^{q} & \alpha^{\prime} x^{\prime} \\
0 & 0 & 0 & 0 & 0 & a
\end{array}\right): a, x, x^{\prime}, y, *, \ldots, * \in K\right\},
$$

where $\alpha, \alpha^{\prime} \in K$ are fixed, and $q=|F|$. As in previous examples, there is one glued component $T_{1}=\{1,2,3,4,5,6\}$, which decomposes with respect to identical gluing as $T_{1}=\{1,2,4,5\} \cup\{3,6\}$. In the equivalence relation of Definition 5.24, $T_{11}$ decomposes into the three equivalence classes: $\gamma_{1}=\{(1,3),(1,5),(2,3),(2,5),(4,5)\}$ (with relative exponent 1 ), $\gamma_{-1}=\{(3,4),(3,6),(5,6)\}$ (with relative exponent -1 ), and the complement $\gamma_{0}$, whose elements are the 13 pairs of relative exponent 0 . (Throughout this example, we use relative exponents as indices.) The relative Frobenius decomposition of a general element, described in equation (6), is

$$
\begin{aligned}
\left(\begin{array}{cccccc}
a & 0 & 0 & * & 0 & * \\
0 & a & 0 & y & 0 & * \\
0 & 0 & a^{q} & 0 & * & 0 \\
0 & 0 & 0 & a & 0 & \alpha \alpha^{\prime} y \\
0 & 0 & 0 & 0 & a^{q} & 0 \\
0 & 0 & 0 & 0 & 0 & a
\end{array}\right)+ & +\left(\begin{array}{cccccc}
0 & 0 & * & 0 & * & 0 \\
0 & 0 & x & 0 & * & 0 \\
0 & 0 & 0 & 0 & 0 & 0 \\
0 & 0 & 0 & 0 & \alpha x & 0 \\
0 & 0 & 0 & 0 & 0 & 0 \\
0 & 0 & 0 & 0 & 0 & 0
\end{array}\right) \\
+ & \left(\begin{array}{cccccc}
0 & 0 & 0 & 0 & 0 & 0 \\
0 & 0 & 0 & 0 & 0 & 0 \\
0 & 0 & 0 & x^{\prime} & 0 & * \\
0 & 0 & 0 & 0 & 0 & 0 \\
0 & 0 & 0 & 0 & 0 & \alpha^{\prime} x^{\prime} \\
0 & 0 & 0 & 0 & 0 & 0
\end{array}\right),
\end{aligned}
$$

and we denote the respective summands in the decomposition of $A$ as $\Gamma_{0}+\Gamma_{1}+\Gamma_{-1}$.

Next, we describe the decomposition corresponding to $t=3$ in Remark 5.32 , The set $T_{1,1,1}$, consisting of $\left(\begin{array}{l}8 \\ 3\end{array}\right)=56$ triples, decomposes into the 7 equivalence classes: $\gamma_{0,0}=[(111)], \gamma_{0,1}=[(113)], \gamma_{1,0}=[(133)], \gamma_{1,-1}=[(134)], \gamma_{0,-1}=$ $[(334)], \gamma_{-1,0}=[(344)]$ and $\gamma_{-1,1}=[(345)]$. Let $\Gamma_{\delta \delta^{\prime}}$ denote the component in (8) corresponding to $\gamma_{\delta \delta^{\prime}}$. Computing via formula (7) we find that $\Gamma_{0,1}=\Gamma_{1,0}=\Gamma_{1}$, that $\Gamma_{0,-1}=\Gamma_{-1,0}=\Gamma_{-1}$, and that $\Gamma_{0,0}=\Gamma_{0}$. On the other hand $\Gamma_{-1,1}=K e_{35}$ and $\Gamma_{1,-1}=K e_{14}+K e_{16}+K e_{26}+K\left(e_{24}+\alpha \alpha^{\prime} e_{46}\right)$ are proper subspaces of previous components; however, we do not get a finer decomposition of $A$. 
Applying the decomposition $A=S+J$, as in equation (5.36), we observe the following. The class $\gamma_{00}=[(111)]$ breaks down to four sub-classes, namely $\gamma_{00}=$ $\left(\gamma_{00}^{=}\right) \cup\left(\gamma_{00}^{=<}\right) \cup\left(\gamma_{00}^{<=}\right) \cup\left(\gamma_{00}^{<<}\right)$, with the obvious interpretation. For example, $\gamma_{00}^{<<}=\{(146),(246)\}$. The corresponding components are $\Gamma_{00}^{=}=S$, the semisimple subalgebra; $\Gamma_{00}^{<<}=K e_{16}+K e_{26} ;$ and $\Gamma_{00}^{=<}=\Gamma_{00}^{<=}=J \cap \Gamma_{00}$. Similar decomposition can be applied to the other classes, although in every case some sub-classes are empty. For example, $\gamma_{0,-1}=\left(\gamma_{0,-1}^{=<}\right) \cup\left(\gamma_{0,-1}^{<<}\right)$, with $\Gamma_{0,-1}^{<<}=K e_{36}$ and $\Gamma_{0,-1}^{=<}=\Gamma_{-1}$.

5.8. Summary. Let $A \subseteq \mathrm{M}_{n}(K)$ be a Zariski-closed $F$-subalgebra, written in Wedderburn block form (Corollary 5.8). In this section we have discussed four useful decompositions. We follow the notation of Definition 5.4 and the idempotents defined in Subsection 5.3

(1) The Peirce decomposition of $A$ is given by $A=\bigoplus \hat{e}_{u} A \hat{e}_{v}$ for $1 \leq u, v \leq k$. Thus every element in $A$ can be written as $a=\sum_{u, v} a_{(u v)}$, with $a_{(u v)} \in A$.

(2) The relative Frobenius decomposition is a finer decomposition of $A$ : Every $a_{(u v)} \in \hat{e}_{u} A \hat{e}_{v}$ decomposes as $a_{(u v)}=\sum_{\gamma} a^{\gamma}$, where $a^{\gamma}$ is defined in (5) and $\gamma \subseteq T_{u, v}$ ranges over the equivalence classes of Definition 5.24. The components $a^{\gamma} \in A$ by Proposition 5.27. This can be refined further by Remark 5.32,

(3) Each idempotent $\hat{e}_{u}$ is a sum of idempotents $\sum_{\mu=1}^{c_{u}} \bar{e}_{u}^{(\mu)}$, as in equation (4), where each idempotent $\bar{e}_{u}^{(\mu)}$ corresponds to the $\mu$-components of identical gluing. The Peirce decomposition of $K A$ is $K A=\sum K A_{u v}^{\left(\mu \mu^{\prime}\right)}$, which gives the decomposition $A=\bigoplus A_{u v}^{\left(\mu \mu^{\prime}\right)}$, with $A_{u v}^{\left(\mu \mu^{\prime}\right)} \subseteq K A$.

If $F$ is infinite, then the decompositions (1), (2) and (3) are identical.

(4) Finally, we have the decomposition $A=\bigoplus A_{r s}$ of matrix blocks, where $A_{r s}=e_{r} A e_{s} \subseteq B$, and $e_{r}$ are the block idempotents defined by the Wedderburn block form. Every $\bar{e}_{u}^{(\mu)}$ breaks down as a sum of such idempotents, as demonstrated in Example 5.21

(5) The decomposition in (4) can be refined by taking equivalence classes of any length $\geq 2$, as described in Remark 5.32 ,

(6) Finally, one may apply the Wedderburn decomposition, or equivalently replace the equivalence classes by fully refined ones of length $\leq \nu$; cf. Subsection 5.7 .

\section{EXPLICIT GENERATORS FOR POLYNOMIAL RELATIONS}

We are ready for a fairly precise description of the polynomial relations of a Zariski-closed algebra $A$, with radical $J$.

Remark 6.1. Suppose $V$ is an $F$-subspace of a $K$-algebra $B$. If $V=V_{1} \oplus V_{2}$ is a direct sum, then any polynomial relation of $V$ is a sum of polynomial relations of $V_{1}$ and $V_{2}$.

In view of this observation, taking the "Wedderburn decomposition" $A=J \oplus S$ inside the Wedderburn decomposition of $B$, we see that the polynomial relations of $A$ are generated by the polynomial relations of $J$ and the polynomial relations of $S$.

The polynomial relations of $S$ come from gluing, which involves $\operatorname{Cent}(S)$, a commutative algebra. 
This leaves the polynomial relations of $J$, whose identifications may be considerably more intricate, especially in the presence of Frobenius gluing. In view of Theorem 4.10, off-diagonal identifications could involve minimal polynomial relations which are $q$-polynomials, i.e., of weak Frobenius type. Denoting the matrix unit in the $(i, j)$ position of the block $B_{r s}$ as $e_{r, s}^{i, j}$, and expanding these to a base of $B$, we have some minimal quasi-linear relations of the form

$$
\sum_{r, s} \sum_{i, j=1}^{n_{r, s}} c_{i j}\left(\lambda_{r, s}^{i, j}\right)^{q_{r, s}}=0
$$

In this case we also say that the gluing has weak Frobenius type. Note that the $q_{r, s}$ are independent of $i$ and $j$, so we might as well assume that $i=j=1$.

Let $\Lambda \subset K\left[\lambda_{1}, \ldots, \lambda_{N}\right]$ denote the set of all weak $F$-Frobenius type polynomials (see Definition 4.9), where $N=\operatorname{dim}_{K}(B)$. Let $\phi$ denote the map given by $a \mapsto a^{q}$ where $q=|F|$ and where, as above, we take $q=1$ if $F$ is infinite. Noting that weak $F$-Frobenius type relations are $F$-linear combinations of $\phi^{j}\left(\lambda_{i}\right)$ for $j \geq 0$ and $i=1, \ldots, m$, we may view $\Lambda$ as a module over $K[\phi]$, under the obvious operation $\phi \cdot a=a^{q}(a \in K)$ and $\phi \cdot \lambda_{i}=\phi\left(\lambda_{i}\right)$. In fact, $\Lambda$ is a free module, spanned by $\lambda_{1}, \ldots, \lambda_{N}$. When $F$ is finite, $K[\phi]$ is isomorphic to the ring of polynomials in one variable over $K$. For infinite $F, K[\phi]=K$, and $\Lambda$ is merely the $K$-dual of $B$, as a vector space.

Theorem 6.2. For any $F$-subspace $A \subseteq B$, the relations poly $A$ form a free module of rank at most $N$ over $K[\phi]$.

Proof. Indeed, poly $A \subseteq \Lambda$ by Theorem 4.10, and $\Lambda$ is a free module over the principal ideal domain $\bar{K}[\phi]$.

We can improve this estimate, by noting that the weak Frobenius relations depend on the Wedderburn block, not on the indeterminate, so we can reduce $\Lambda$ to the module generated by one representative indeterminate for each Wedderburn block above the diagonal. This is $\left(\begin{array}{c}m \\ 2\end{array}\right)$, where $m$ is the number of diagonal Wedderburn blocks in the given representation of $A$ (clearly $\left.m^{2} \leq N\right)$. Thus, we have

Corollary 6.3. The weak Frobenius relations of a Zariski-closed F-subalgebra A of $B$ can be defined by at most $\left(\begin{array}{c}m \\ 2\end{array}\right)$ polynomial relations, where each relation is duplicated $\operatorname{dim}\left(B_{u v}\right)$ times. (For example, one needs four relations to define $\mathrm{M}_{2}\left(\mathbb{F}_{q}\right)$ inside the algebraic closure: $\lambda_{i j}^{q}=\lambda_{i j}$ for $i, j=1,2$.)

We can improve this result even further.

\section{Lemma 6.4.}

(1) In a polynomial relation of weak Frobenius type, we may assume that one of the $q_{i j}=1$.

(2) Given two polynomial relations $f_{1}, f_{2}$ of weak Frobenius type and given some $\lambda_{i}$, we may modify these two polynomial relations to assume that $\lambda_{i}$ appears in at most one of them.

Proof. (1) Otherwise we can write each $q_{i j}=q q_{i j}^{\prime}$, and then

$$
\left(\sum_{i=1}^{m} \sum_{j \geq 1} c_{i j} \lambda_{i}^{q_{i j}^{\prime}}\right)^{q}=\sum_{i=1}^{m} \sum_{j \geq 1} c_{i j} \lambda_{i}^{q_{i j}}=0,
$$


so taking the $q$-root yields a polynomial relation of lower degree, and we conclude by induction.

(2) The argument is by induction on the degree $q^{d_{i}}$ of $\lambda_{i}$ in $f_{1}$ and $f_{2}$. Suppose $d_{1} \geq d_{2}$. Then the degree of $q$ in $f_{1}-f_{2}^{d_{1}-d_{2}}$ is less than $d_{1}$, so we continue by induction.

Corollary 6.5. Suppose the weak Frobenius relations of $A$ are defined by $\mu \leq\left(\begin{array}{c}m \\ 2\end{array}\right)$ polynomial relations $\left\{f_{1}, \ldots, f_{\mu}\right\}$. Then we may take $\mu$ variables and assume that each of them appears in at most one of $\left\{f_{1}, \ldots, f_{\mu}\right\}$.

\section{Generic Zariski-Closed algebras and PI-Generic Rank}

Our study of Zariski-closed algebras has been motivated by the desire to find an algebra in a given variety whose structure is especially malleable. Since every variety contains a relatively free algebra, we are led to study the relatively free algebras in the variety generated by Zariski-closed algebras. These are the generic algebras, which can be described in terms of "generic" elements. First we start with the classical setting, and then we see how the presence of finite fields complicates the situation and requires a more intricate description.

We want to define a "finitely generated" generic algebra. This means that we need to consider polynomial identities in only a finite number of indeterminates. First, we need to clarify exactly what we mean generically by "generation."

Definition 7.1. The topological rank of a Zariski-closed algebra $A$ is defined as the minimal possible number of generators of an $F$-subalgebra $A_{0}$ of $A$ for which $A_{0}^{\mathrm{cl}}=A$.

Remark 7.2. By Theorem 3.28, every semiprime Zariski-closed algebra is a finite direct sum of simple algebras, and thus has finite topological rank.

Thus, the obstruction to finite topological rank is found in the radical.

Example 7.3. Let $K$ be an infinite dimensional field extension of a finite field $F$, and consider

$$
A=\left(\begin{array}{cc}
F & K \\
0 & F
\end{array}\right) .
$$

This Zariski-closed algebra has infinite topological rank, since any finite number of elements generates only a finite subspace of $K$ in the 1,2 position, which is Zariski-closed.

Nevertheless, we do have the following information.

Remark 7.4. When the Peirce idempotent $\tilde{e}$ has infinite type (cf. Definition [5.18), then the spaces $A \tilde{e}$ and $\tilde{e} A$ have finite topological rank, since they are naturally vector spaces over $K$ (although this is not the structure of the initial vector space). The action is via the isomorphism of $K$ with the center of the prime component corresponding to $\tilde{e}$.

7.1. Generic algebras over an infinite field. Clearly, the topological rank of an f.d. algebra $A$ over field $K$ is not greater than its dimension, which is given to be finite. Thus, in this case, we need only finitely many elements to define the generic algebra. 
Construction 7.5. The classical construction of a generic algebra of an f.d. algebra $A$ over an infinite field $F$ is to take a base $b_{1}, \ldots, b_{n}$ of $A$ over $F$, adjoin indeterminates $\xi_{i}^{(k)}$ to $F(i=1, \ldots, n, k \in \mathbb{N})$, and let $A^{\prime}$ be the algebra generated by the "generic" elements $Y_{k}=\sum_{i=1}^{n} \xi_{i}^{(k)} b_{i}, k \in \mathbb{N}$. It is easy to see BR, Example $3.26]$ that in the case where $F$ is infinite, $A^{\prime}$ is PI-equivalent to $A$, and in fact $A^{\prime}$ is relatively free in the variety defined by $\operatorname{id}(A)$. The most celebrated example in PI-theory is when $A=\mathrm{M}_{n}(F)$, the algebra of $n \times n$ matrices. Then $A^{\prime}$ is the algebra of generic $n \times n$ matrices, generated by the generic matrices $Y_{k}=\left(\xi_{i j}^{(k)}\right)_{i j}, k \in \mathbb{N}$.

Example 7.6. The generic upper triangular matrix $Y_{k}=\left(\begin{array}{cc}\xi_{1}^{(k)} & \xi_{2}^{(k)} \\ 0 & \xi_{3}^{(k)}\end{array}\right)$ is defined over the polynomial algebra $C=F\left[\xi_{j}^{(k)}: j=1,2,3, k \in \mathbb{N}\right]$. We get the generic algebra of upper triangular matrices by taking the subalgebra of $\mathrm{M}_{n}(C)$ generated by the $Y_{k}$.

Construction 7.7. Alternatively, when building generic elements for an arbitrary f.d. algebra $A$ over an infinite field, we could take the powers of the radical $J$ into account. Writing $A=S \oplus J$ by Theorem 3.33, where $J^{\nu}=0$, we can view $J^{2}$ as those blocks at least two steps above the diagonal, i.e., lying in $\sum_{r+2 \leq s} B_{r s}$. We take a generic algebra for $S$ and generic elements for $J / J^{2}$ (which can be viewed as a complementary subspace for $J^{2}$ inside $J$ ), taking identical gluing into account; these then yield generic elements for $A$. This also will be the approach that we take for Zariski-closed algebras over arbitrary fields.

7.2. PI-generic rank over an arbitrary field. Since the topological rank could be infinite, we look for an alternative concept which is more closely relevant to PI-theory.

Definition 7.8. The PI-generic rank of $A$ is the minimal number $m$ of elements needed to generate a subalgebra satisfying the same PI's as $A$. Then the relatively free PI-algebra of $A$ could also be generated by $m$ elements. In the literature, the PI-generic rank is often called the basic rank.

Clearly, the PI-generic rank is less than or equal to the topological rank.

\section{Example 7.9.}

$$
A=\left\{\left(\begin{array}{ccc}
\alpha & \beta & \gamma \\
0 & \alpha & \beta \\
0 & 0 & \alpha
\end{array}\right): \alpha \in F, \beta \in K\right\}
$$

is a commutative algebra, having PI-generic rank 1, but having infinite topological rank when $K$ is infinite dimensional over $F$. This example also shows that gluing can lower the PI-rank.

When computing PI-generic rank, we study a polynomial in terms of substitutions of its monomials, as usual, which can be complicated by the absence of indeterminates in certain monomials. Accordingly, recall from [R1, Definition 2.3.15] that a polynomial $f$ is called blended if each monomial appearing in $f$ must appear in each monomial of $f$. As noted in [R1, Exercise 2.3.7], any PI is a sum of blended PI's, seen by specializing each indeterminate to 0 in turn. Thus, we can limit ourselves to blended PI's when determining the PI-generic rank. 
Let us consider the PI-generic rank of a Zariski-closed PI-algebra. Although when $F$ is infinite, this is obviously finite (since the variety contains a finite dimensional algebra), the situation becomes much more interesting when $F$ is finite. Although, as already seen in Example 7.3, we might need infinitely many generic elements to generate our algebra, we aim to show, however, that the PI-generic rank is always finite.

Theorem 7.10. Any Zariski-closed algebra A (over an arbitrary field) has finite PI-generic rank.

Proof. (As noted above this statement is trivial for algebras over infinite fields.) We decompose $A=S \oplus J$, where $S$ is semisimple and $J$ is nilpotent, of nilpotence index $\nu$. By Proposition 2.8, it is enough to consider specializations for which every variable takes values either in $S$ or in $J$.

We only need to consider blended polynomials. In any non-zero evaluation of a polynomial on $A$, at most $\nu-1$ components can belong to $J_{1}$, as defined in Remark 5.36. But $J_{1}$ being a variety, has a finite number $\psi$ of irreducible components, each of which has a generic element which we can use for the radical substitution. Our "generic" radical substitution could be taken to be the sum of these substitutions.

This leaves the semisimple substitutions, which we consider "layered" around the radical substitutions. The PI-generic rank of $S$ is less than or equal to its topological rank, which is finite; cf. Remark 7.2 .

In view of $A=S \oplus J$, we see that the PI-generic rank is at most $\mu+\nu-1$, where $\mu$ is the PI-rank of $S=A / J$.

By passing to the Zariski closure, we have:

Corollary 7.11. Any representable algebra A (over an arbitrary field) has a PIequivalent algebra with finite PI-generic rank.

Remark 7.12. We can improve the bound given in Theorem 7.10, First, any central simple algebra over an infinite field is generated by two elements; thus, its topological rank (and thus PI-generic rank) is 2 . Thus, when $F$ is infinite, $\mu=2$, so our bound becomes $\nu+1$. (This can be lowered even further, since the Zariski closure of a one-generator algebra contains its radical part.)

Over a finite field $F$, any simple algebra has the form $\mathrm{M}_{n}(F)$. If $|F|>n$, then $\mathrm{M}_{n}(F)$ is generated by two elements, one being the diagonal with distinct entries and the other being the upper triangular matrix $\sum_{i=1}^{n-1} e_{i, i+1}$. On the other hand, when $|F|^{2}<n$, the topological rank starts growing, because of repeating eigenvalues, although obviously the topological rank is finite (bounded by $n^{2}$ ) and in fact grows much more slowly, bounded by $2+\log _{|F|} n$, as seen by the argument given above. At any rate, the topological rank, and thus the PI-rank, of any central simple algebra of dimension $n^{2}$ is finite, bounded by some function of $n$. Arguing by components shows that the PI-generic rank of any non-commutative semisimple algebra is given along the same lines.

When $F$ is a finite field of $q$ elements, there are only finitely many possible elements in $\mathrm{M}_{n}(F)$, namely $q^{n^{2}}$, and thus $q^{2 n^{2}}$ possible ordered pairs. If we have more components in $A$, some pair must repeat itself, and thus the corresponding components become glued when $\psi \geq q^{n^{2}}$ unless we have a greater number of generators. 
Thus the size of $\psi$ can also force up (logarithmically) the bound for the PI-generic rank.

7.3. Generic representable algebras, not over an infinite field. Our main theorem in this section is that for any representable algebra $A$ there exists a relatively free, finitely generated algebra in the variety $\operatorname{Var}(A)$ obtained by the identities of $A$.

Although, for any representable algebra $A$ over an infinite field $F$, the classical construction of a generic algebra for $A$ is PI-equivalent to the original algebra $A$, this is no longer the case when $F$ is finite (or even worse, when there is no base field). Thus, when considering finite characteristic, we need to introduce new commutative rings that need not be fields.

Example 7.13. Suppose the field $F$ contains $q$ elements. Then $F$ is not PIequivalent to the ring of polynomials $F[\xi]$, so we must pass to $F[\xi] /\left\langle\xi^{q}-\xi\right\rangle$, where the image $\bar{\xi}$ of $\xi$ is a generic element. Note that $F[\xi] /\left\langle\xi^{q}-\xi\right\rangle$ is isomorphic to a direct product of $q$ copies of $F$, so another way of viewing our generic element is as a $q$-tuple listing the elements of $F$. Unfortunately, this may not suffice to describe the PI's since they involve more than one substitution. For two generic elements we need to pass to

$$
F\left[\xi_{1}, \xi_{2}\right] /\left\langle\xi_{1}^{q}-\xi_{1}, \xi_{2}^{q}-\xi_{2}\right\rangle,
$$

which is isomorphic to a direct product of $q^{2}$ copies of $F$, and so on. Of course, since the identity of commutativity only requires two variables, this is enough for the generic element of the variety of $F$, but we already see the difficulty arising of predicting how many generic elements we need to construct the generic algebra.

Nevertheless, there is a way to define the generic algebra for a general Zariskiclosed algebra $A=A^{\text {cl }}$ (represented in some f.d. algebra $B$ over an algebraically closed field $K$ ). The idea is to define everything as generically as possible.

Construction 7.14 (General construction of generic algebras). Letting $\mathcal{C}_{1}, \ldots, \mathcal{C}_{t}$ denote the irreducible components of $A^{\mathrm{cl}}$ under the Zariski topology, suppose each $\mathcal{C}_{i}$ is defined over a field with $q_{i}$ elements. Then we need $s$ "mutually generic" elements $b_{i 1}, \ldots, b_{i s}$ in each component. Towards this end, we take a generic element

$$
b \in \mathcal{C}_{1}^{s} \times \cdots \times \mathcal{C}_{t}^{s},
$$

where each $\mathcal{C}_{i}^{s}$ denotes the direct product of $s$ copies of $\mathcal{C}_{i}$. Thus $b$ has the form $\left(\left(b_{11}, \ldots, b_{1 s}\right),\left(b_{21}, \ldots, b_{2 s}\right), \ldots,\left(b_{\mu 1}, \ldots, b_{\mu s}\right)\right)$, where each $\left(b_{i 1}, \ldots, b_{i s}\right) \in \mathcal{C}_{i}$; by definition, the $b_{i k}$ are "mutually generic". Next, we define the generic coefficient ring

$$
C=F\left[\xi_{i k}: 1 \leq i \leq s, 1 \leq k \leq \mu\right] /\left\langle\xi_{i k}^{d_{i}}-\xi_{i k}: 1 \leq i \leq s, 1 \leq k \leq \mu\right\rangle,
$$

and the generic elements $Y_{k}=\sum_{i=1}^{s} \bar{\xi}_{i k} b_{i k}(k=1, \ldots, \mu)$, where $\bar{\xi}_{i k}$ is the image of $\xi_{i k}$ in $C$. The subalgebra of $B$ generated by the $Y_{k}$ serves as our generic algebra for the variety generated by $A$.

Note that this construction is completely general since the free algebra of any variety is representable; however, this is difficult to prove (using the theory developed in this paper).

Theorem 7.15. The algebra $\mathcal{Y}=F\left\{Y_{1}, \ldots, Y_{\mu}\right\}$ of Construction 7.14 is relatively free in $\operatorname{Var}_{\mathrm{F}}(A)$, with free generators $Y_{1}, \ldots, Y_{\mu}$. If $t \geq$ PI-rank $A$, then $\operatorname{Var}_{\mathrm{F}}(\mathcal{Y})=$ $\operatorname{Var}_{\mathrm{F}}(A)$. 
Proof. Any set of mutually generic elements specialize to arbitrary elements of $A$, so it remains to show that $\mathcal{Y}$ satisfies the identities of $A$. But this is clear, since any element is the sum of generic components, which satisfy the identities of $A$ by definition.

This construction also works for non-associative Zariski-closed algebras of arbitrary signature, in the framework of universal algebra.

Remark 7.16. One cannot simply describe the generic algebra in Construction 7.14 by taking the $b_{i}$ from a base of the extended algebra as in Construction 7.5 because the Gel'fand-Kirillov (GK) dimensions do not match, as evidenced by Belov's computation discussed in Remark 5.6. Indeed, the generic coefficient ring $C$ is finite, and thus the GK dimension would only be equal to the GK dimension of the first block, instead of the sum of the GK dimensions of the blocks.

Example 7.17. Let $A$ be the algebra of triangular matrices with entries as follows: $\left(\begin{array}{ll}\alpha & \beta \\ 0 & \gamma\end{array}\right)$, where $\alpha$ is in the finite field $F$, and $\beta, \gamma$ are in an infinite field extension $K$ of $F$. Continuing the notation of Construction 7.14, the generic algebra is generated by matrices of the form $Y_{i}=\left(\begin{array}{cc}\xi_{i 1} & \xi_{i 2} \\ 0 & \xi_{i 3}\end{array}\right)$, where $\xi_{i 1}$ is a generic element of $C$, whereas $\xi_{i 2}, \xi_{i 3}$ are indeterminates over $K$.

Example 7.18. The generic upper triangular matrices for an algebra with Frobenius gluing of power $q$ along the diagonal can be written in the form $Y_{k}=\left(\begin{array}{cc}\xi_{1 k} & \xi_{2 k} \\ 0 & \xi_{1 k}^{q}\end{array}\right)$.

When Frobenius gluing is involved, we can still use the generic coordinate ring of Construction 7.14. The generic description of partial gluing up to infinitesimals becomes more complicated in the presence of Frobenius gluing, because we need to deal both with the Frobenius automorphism and also with the degree of the infinitesimal.

We close by providing an explicit construction for generic PI-algebras of Zariskiclosed algebras. As in Construction 7.7, we take the powers of the radical $J$ into account.

Construction 7.19 (The explicit generic algebra of a Zariski-closed algebra of finite topological rank). In view of Construction [7.5, we may assume the base field $F$ is finite. The construction requires generic elements for the Zariski-closed algebra $A$, which will be defined over the ring of polynomials $F\left[\xi_{1}, \xi_{2}, \ldots\right]$ (with an appropriate indexing). There are several methods; we choose the one that is perhaps most intuitive according to the structure, but rather intricate. Our point of departure is the Wedderburn Block form (Theorem 5.7). Namely, write $A \subset \mathrm{M}_{n}(K)$, where $F \subseteq K$.

(1) First consider the center $A_{0}$ of a simple component in $A$, such as

$$
A_{0}=\left\{\left(\begin{array}{cc}
\alpha & 0 \\
0 & \alpha^{p}
\end{array}\right): \alpha \in K\right\}
$$

This algebra may be described by the Frobenius gluing of $1 \times 1$ blocks along a single gluing component. Namely, the algebra has the form $\left\{\sum_{i=1}^{s} \alpha^{\phi_{i}} e_{i}\right.$ : $\alpha \in K\}$, where $e_{i}$ are the basic idempotents and $\phi$ is the exponent vector, taking $q$-power values (for $q=|F|$ ). The generic elements can be taken as $X_{k}=\sum_{i=1}^{s} \xi_{k}^{\phi_{i}} e_{i}$. 
(2) Let $S$ be a simple component of $A$. In $B \subseteq \mathrm{M}_{n}(K), S$ is contained in a direct sum of matrix blocks of the same size. Let $e_{i}^{j j^{\prime}}$ denote the $1 \times 1$ matrix units in the $i^{\text {th }}$ block, whose corresponding block idempotent is $e_{i}$. Keeping the notation as above, the generic elements can be taken as $X^{k, j j^{\prime}}=\sum_{i=1}^{s}\left(\xi_{k}^{j j^{\prime}}\right)^{\phi_{i}} e_{i}^{j j^{\prime}}$, where the variables are $\xi_{k}^{j j^{\prime}}$. For example, we could have

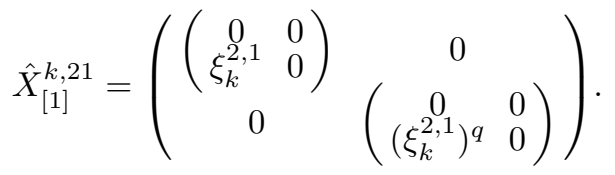

(3) Let $d$ denote the number of glued components in $A$, and let $\hat{e}_{u}$ denote the idempotent corresponding to the $u^{\text {th }}$ component, decomposed as $\hat{e}_{u}=$ $\sum_{r \in T_{u}} e_{r}$, as in Subsection [5.3. Let $\hat{X}_{[u]}^{k, j j^{\prime}}$ denote the glued sum of appropriate powers of $\xi_{k}^{j j^{\prime}}$, placed in the $\left(j, j^{\prime}\right)$ entry of the glued blocks, where the sum is over the blocks $r \in T_{u}$. Each generic element of this type can be decomposed as a sum $\hat{X}_{[u]}^{k, j j^{\prime}}=\sum_{r \in T_{u}} X_{r}^{k, j j^{\prime}}$, where $X_{r}^{k, j j^{\prime}}$ is the appropriate power of $\xi_{k}^{j j^{\prime}}$, placed in the $\left(j, j^{\prime}\right)$ entry of the $r^{\text {th }}$ block. The $X_{[u]}^{k, j j^{\prime}}$ are the semisimple part of our generic elements.

Let $b_{1}, \ldots, b_{\tau}$ be a topological basis for $S$. We continue as in Remark 5.32. For every $2 \leq t \leq \nu$ (where $\nu$ is the nilpotence index of $J$; see Proposition 5.38), for all indices $1 \leq u_{1}, \ldots, u_{t} \leq d$, and for every (fully refined) equivalence class $\gamma \subseteq T_{u_{1}, \ldots, u_{t}}$ (see Definition 5.24 and Remark 5.36), we take all the elements

$$
X_{\vec{k}}^{*, \gamma}=\sum_{\left(r_{1}, \ldots, r_{t}\right) \in \gamma} X_{r_{1}}^{k_{1}, j_{1} j_{1}^{\prime}} b_{p_{1}} X_{r_{2}}^{k_{2}, j_{2} j_{2}^{\prime}} \cdots X_{r_{t-1}}^{k_{t-1}, j_{t-1} j_{t-1}^{\prime}} b_{p_{t-1}} X_{r_{t}}^{k_{t}, j_{t} j_{t}^{\prime}},
$$

where each of $p_{1}, \ldots, p_{t}$ ranges over the values 1 through $\tau$, each $k_{i}$ ranges over $\mathbb{N}$, and each $\left(j_{i}, j_{i}^{\prime}\right)$ ranges over the matrix entries of the blocks in the $r_{i}$ place (they have the same dimensions for each $\left(r_{1}, \ldots, r_{t}\right) \in \gamma$ ).

Theorem 7.20. The algebra $\mathcal{Y}=F\left\{X_{\vec{k}}^{*, \gamma}: k_{1}, \ldots, k_{t} \leq \mu\right\}$ of Construction 7.19 (where we range over all possible choices of $t, p_{1}, \ldots, p_{t}$ and $j_{1}, j_{1}^{\prime}, \ldots, j_{t}, j_{t}^{\prime}$ and $\gamma)$ is relatively free in $\operatorname{Var}_{\mathrm{F}}(A)$, with free generators $\tilde{X}_{\vec{k}}^{*, \gamma}$. If $\mu \geq \mathrm{PI}-\mathrm{rank} A$ (in particular, if $\mu$ is at least the number of topological generators of $A$ ), then $\operatorname{Var}_{\mathrm{F}}(\mathcal{Y})=\operatorname{Var}_{\mathrm{F}}(A)$.

Proof. Same as Theorem 7.15 .

Corollary 7.21. For any representable algebra $A$, its variety has a finitely generated relatively free algebra.

Proof. In view of Theorem 7.10, we may assume that $A$ has finite PI-rank, and thus we are done by Theorem 7.20 .

This construction is needed in a forthcoming paper, where we describe varieties of algebras in terms of a certain kind of quiver. 


\section{REFERENCES}

[B1] Belov, A. Ya., Algebras with polynomial identities: Representations and combinatorical methods, Doctor of Science Dissertation, Moscow (2002).

[B2] Belov, A., Counterexamples to the Specht problem, Sb. Math. 191 (2000), pp. 329-340. MR.1773251 (2001g:16043)

[BR] Belov, A.Ya. and Rowen, L.H. "Computational aspects of polynomial identities". Research Notes in Mathematics, 9. AK Peters, Ltd., Wellesley, MA, 2005. MR 2124127 (2006b:16001)

[GZ] Giambruno, A. and Zaicev, M., Minimal varieties of algebras of exponential growth, Adv. Math. 174 (2003), pp. 310-323. MR1963697(2004b:16028)

[H] Humphreys, J., Linear algebraic groups, Springer Lecture Notes in Mathematics 21 Springer, New York, 1975. MR0396773 (53:633)

[KMT] Kambayashi, T.; Miyanishi, M. and Takeuchi, M., "Unipotent algebraic groups", Lecture Notes in Mathematics, 414, Springer-Verlag, Berlin-New York, 1974. MR0376696 $(51: 12871)$

[L] Lewin, J., A matrix representation for associative algebras. I and II, Trans. Amer. Math. Soc. 188 (2) (1974), 293-317; ibid. MR0338081 (49:2848)

[M] Miyanishi, M., Questions of rationality of solvable algebraic groups over non-perfect fields, Annali Mat. Pura Appl. 61(4), (1963) 97-120. MR0158891 (28:2113)

[P] Putcha, M., Linear algebraic monoids, London Math. Soc. Lecture Notes 133, Cambridge University Press, 1988. MR964690 (90a:20003)

[R1] Rowen, L.H., Polynomial Identities in Ring Theory, Academic Press, Inc., Pure and Applied Math., 84, New York, 1980. MR576061 (82a:16021)

[R2] L Ring Theory I, Pure and Applied Math, 127, Academic Press., Inc., New York, 1988. MR $1095047(94 \mathrm{e}: 16001)$

[R3] Graduate Algebra: A noncommutative view, Graduate Series in Math., Amer. Math. Soc., Providence, RI, 2008. MR2462400 (2009k:16001)

[T] Tits, J., "Lectures on algebraic groups", Dept. of Math., Yale Univ. - New Haven, 1966/67.

Department of Mathematics, Bar-Ilan University, Ramat-Gan 52900, Israel

E-mail address: belova@macs.biu.ac.il

Department of Mathematics, Bar-Ilan University, Ramat-Gan 52900, Israel

E-mail address: rowen@macs.biu.ac.il

Department of Mathematics, Bar-Ilan University, Ramat-Gan 52900, Israel

E-mail address: vishne@macs.biu.ac.il 